\title{
9 Interleavers
}

René M. de Ridder and Chris G. H. Roeloffzen

\subsection{Introduction}

In an optical communication system using wavelength division multiplexing (WDM), information is transmitted over several "channels", each at a different optical wavelength $\lambda_{i}$ (or optical carrier frequency $f_{i}$ ). An interleaver, also known as a slicer, is an optical filter having at least one input and two complementary outputs, with an optical transfer function that is periodic in frequency. In this way, for example, even-numbered optical channels can be routed to one output port while the odd-numbered channels will emerge from the other output port, as illustrated in Fig. 9.1. Such an interleaver can also be used the other way round, i. e. for combining two "combs" of optical channels, one shifted by half a channel spacing with respect to the other, into a single comb with half the channel spacing.

Besides the basic $1 \times 2$ interleaver function illustrated in Fig. 9.1, more complicated configurations may be used, as shown in Fig. 9.2.

In principle, any optical (de)multiplexer having a periodic response with frequency may be used as an interleaver, for example arrayed waveguide gratings (AWG, cf. Chap. 4), Fabry-Perot resonators (Chap. 6) or ring resonators (Chap. 8). An overview of interleaver technology with an emphasis on bulk crystal optics is given by Cao et al. [1]. Interleavers that are applied in optical telecommunications should have a frequency-periodic

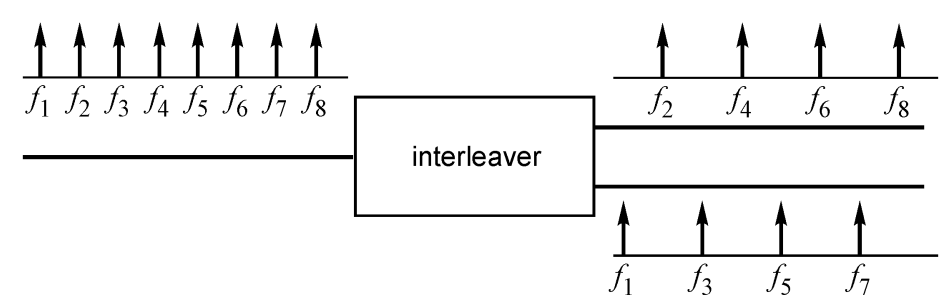

Fig. 9.1. Basic operation of an $1 \times 2$ interleaver as a frequency demultiplexer 


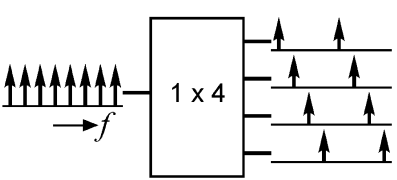

(a)

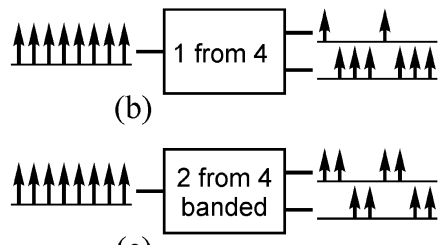

(c)

Fig. 9.2. Some examples of other interleaver configurations

response, because the ITU grid specifying the optical channels defines a set of equidistant optical frequencies (cf. Appendix, Sect. A.1). Despite this, these devices are sometimes referred to as wavelength slicers or wavelength interleavers. (If an interleaver is periodic in frequency it will not be strictly periodic in wavelength since $\lambda=c / f$, with $c$ the vacuum speed of light.) If needed, however, strictly wavelength periodic interleavers can be designed [2].

The main applications of interleavers are in wavelength routing, (de)multiplexing, and pre-filtering. In the latter application, the interleaver is used as a first filtering stage which should have a transfer function approximating a rectangular shape as much as possible. The optical channels at its output ports still have the same bandwidth as before, but they are separated farther apart, thus making further routing or filtering operations less demanding. As an example, Fig. 9.3 shows the application of an interleaver for upgrading existing network nodes with $100 \mathrm{GHz}$ channel spacing to ones with $50 \mathrm{GHz}$ channel spacing. An additional advantage of integrating an interleaver with an AWG is the possibility of improving the AWG passband shape [3].

Figure 9.4 shows how a full demultiplexer can be built by cascading interleavers, in a binary tree structure, each stage having twice the free spectral

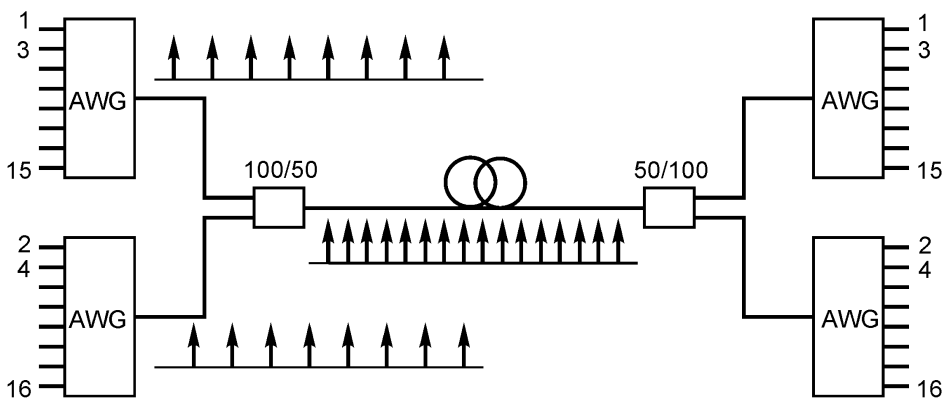

Fig. 9.3. 50/100 GHz interleaver as last-stage multiplexer and first-stage demultiplexer, combined with $100 \mathrm{GHz}$ arrayed waveguide grating (AWG) (de)multiplexers to form a $50 \mathrm{GHz}$ channel spacing system 


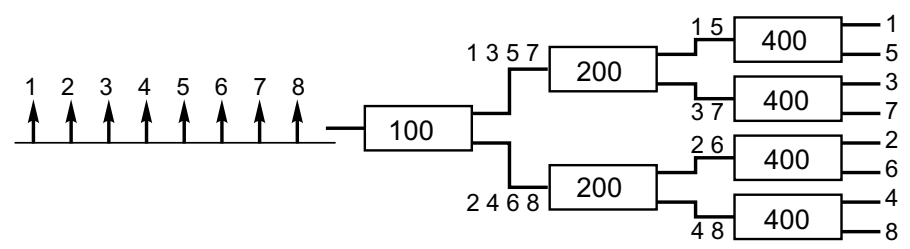

Fig. 9.4. Full binary tree 8 -channel demultiplexer based on cascaded $1 \times 2$ interleavers. Eight channels with $50 \mathrm{GHz}$ optical carrier frequency spacing are assumed, numbered $1-8$. The numbers $100,200,400$, written in the interleaver symbols of this example indicate the free spectral range (the frequency period in $\mathrm{GHz}$ ) of these components, resulting in a $50 \mathrm{GHz}$ input channel spacing

range (the period of the frequency response) as the preceding one, e. g. [4]. Finally, Fig. 9.5 illustrates the principle of an add-drop multiplexer built from two partial binary trees [5].

The obvious choice of a physical principle for implementing interleavers is interference, since this phenomenon has an inherently frequency-periodic nature. The interleaver operation can be demonstrated using an asymmetric Mach-Zehnder interferometer (MZI).

The MZI is one of the most important building blocks in (integrated) optics. It is widely applied for such functions as switching, routing, and modulating optical signals, and it is a basic structure for many optical sensors. A schematic drawing of an MZI is shown in Fig. 9.6. Although MZI's can be implemented in several ways (e.g. with beam splitters and mirrors in freespace; using crystal optics; or in optical fibres), here we consider a structure based on planar optical waveguides. It consists of two 3-dB couplers connected by two single-mode optical channel waveguides, where the signal in one path experiences a phase delay of $\Delta \varphi$ with respect to the other.

The couplers, which can be for example directional couplers, split the optical input power entering one of its input channels into two equal power output signals (each $50 \%$ or $-3 \mathrm{~dB}$ of the input power), having $90^{\circ}$ optical phase difference. For symmetry reasons, a signal in the other input would lead to two equal output signals having $-90^{\circ}$ phase difference. Since the

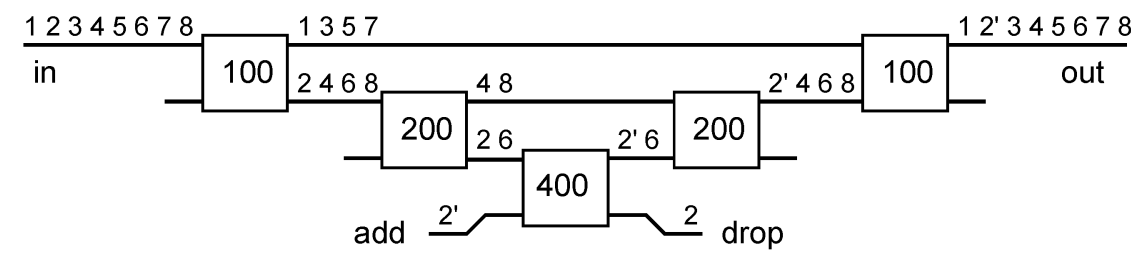

Fig. 9.5. Binary tree cascaded $2 \times 2$ interleavers organised to form a 1 from 8 add-drop multiplexer 


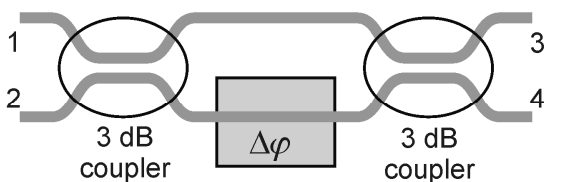

(a)

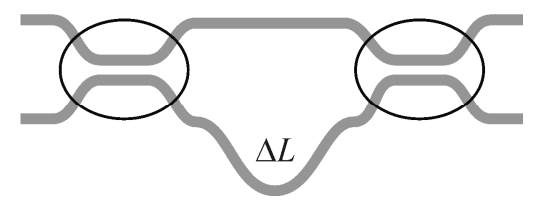

(b)

Fig. 9.6. Mach-Zehnder interferometer (MZI). (a) Basic structure. (b) Asymmetric MZI

coupler is reciprocal, two equal power coherent input signals with $90^{\circ}$ phase difference would combine into a single output signal at one output channel, while $-90^{\circ}$ phase difference would direct all power to the other output channel. With two in-phase coherent input signals, each output channel would carry half of the total available power. Hence, it is obvious that the phase difference determines the power distribution over the output channels. It can be shown that the signals at the output ports of the MZI are related to those at the input ports as

$$
\begin{aligned}
& P_{3}=P_{1} \sin ^{2} \frac{\Delta \varphi}{2}+P_{2} \cos ^{2} \frac{\Delta \varphi}{2} \\
& P_{4}=P_{1} \cos ^{2} \frac{\Delta \varphi}{2}+P_{2} \sin ^{2} \frac{\Delta \varphi}{2}
\end{aligned}
$$

where $P_{i}$ is the signal power in port $i$, as indicated in Fig. 9.6.

The phase difference $\Delta \varphi$ can be caused by a differential propagation delay in the interferometer arms due to a length difference $\Delta L$ between the arms, as illustrated in Fig. 9.6b,

$$
\Delta \varphi=\beta \Delta L=\frac{2 \pi}{c} f n_{\text {eff }} \Delta L
$$

where $\beta$ is the propagation constant of the mode in the delay section, $f$ is the frequency of the wave, $n_{\text {eff }}$ is the effective refractive index of the waveguide mode, and $c$ is the speed of light in vacuum. If the dispersion is neglected ( $n_{\text {eff }}$ is assumed to be constant), the phase difference is proportional to both the frequency $f$ and the delay length $\Delta L$. Combining (9.1) and (9.2), the power transfer from port 1 to ports 3 and 4 as a function of $f$ is depicted in Fig. 9.7. The free spectral range (FSR), which is the period $\Delta f_{\mathrm{FSR}}$ in the frequency response is given by

$$
\Delta f_{\mathrm{FSR}}=\frac{c}{n_{\text {eff }} \Delta L}
$$

Figure 9.7 shows that a comb of optical signal frequencies with a spacing of $\Delta f=\Delta f_{\mathrm{FSR}} / 2$ may be split into two combs, each having the double frequency spacing. 


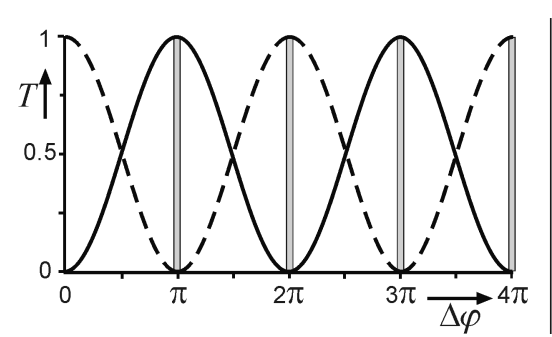

(a)

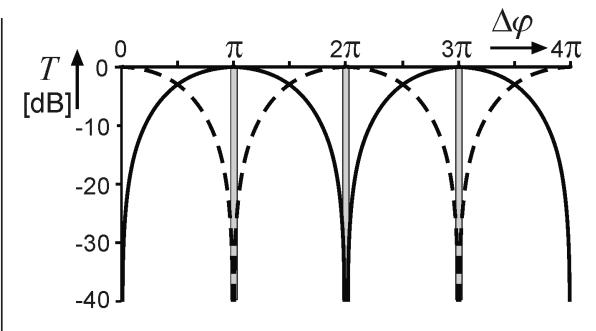

(b)

Fig. 9.7. Normalised power transfer functions of an asymmetric MZI; the solid curve indicates $P_{3} / P_{1}$, while the dashed curve indicates $P_{4} / P_{1}$. (a) Linear power scale; (b) logarithmic scale. The grey vertical bars indicate the optical signal channels which are routed from port 1 alternately to ports 3 and 4 (de-interleaving)

As may be obvious from Fig. 9.7, an MZI does not have an ideal rectangular-shaped transfer function. It does not provide sufficient tolerance for wavelength deviations, laser linewidth and modulation bandwidth, which should typically be about $40 \%$ of the grid spacing. Especially the suppression of unwanted channels is critical $(25 \mathrm{~dB}$ is a common requirement, leading to a useful bandwidth of only $8 \%$ of the channel spacing for an MZI, see the narrow channel bars in Fig. 9.7).

One method for improving the transfer function comes from the observation that a periodic function with "arbitrary" shape can be composed like a Fourier series, from a set of harmonically related sinusoidal functions which are added together with appropriate amplitude and phase. Figure 9.8a shows the conceptual picture, where multiple delayed copies of the input signal are combined into a weighted sum. For a periodic filter response, the delays $\tau_{i}$ should be integer multiples of the unit delay $\tau_{1}$ defining the FSR.

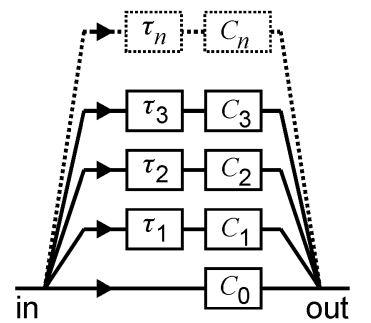

(a)

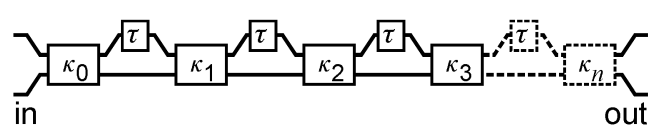

(b)

Fig. 9.8. Composite filters. (a) Multiple parallel weighted delay-line paths; for a Fourier-type synthesis, all $\tau_{i}$ should be an integer multiple of the smallest delay, $\tau_{1}$. (b) Equivalent lattice filter, with $\tau=\tau_{1}$ 
For approximating a "rectangular" filter transfer function only odd multiples of $\tau_{1}$ are needed. The weighting constants $C_{i}$ are the Fourier coefficients. Since it turns out to be rather difficult to realise low loss multiport optical splitters and combiners, the equivalent approach shown in Fig. $9.8 \mathrm{~b}$, requiring only $2 \times 2$-type couplers is preferred. This type of structure is known as a lattice filter or resonant coupler. The equivalence with the parallel delay line filter may be intuitively understood by considering all possible paths that an optical signal can take through the lattice. The output signal can then be written as the sum of a number of terms, each involving an integer $(0,1, . . n)$ multiple of the unit delay $\tau$. A possible implementation, illustrated in Fig. 9.9, is by concatenation of MZI-like elements, using couplers with specific (non 50-50) power coupling ratios $\kappa_{i}$. In addition to the fixed path length differences $\Delta L_{j}$ generating the frequency-periodic response, in general also tuneable phase-shifters $\Delta \varphi_{k}$ are implemented in each MZI-section for fine-tuning purposes. Several design methods have been discussed in the literature, e. g. [2,6-14]. This type of interleaver is analysed in detail in Sect. 9.3.1.

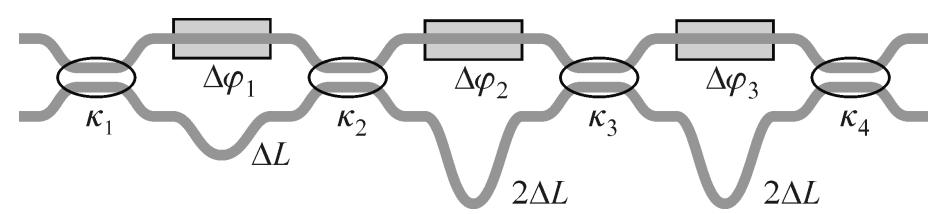

Fig. 9.9. Band flattening: lattice-type filter of concatenated MZI-like structures. The double delay length sections $(2 \Delta L)$ arise from combining two sections where the coupling ratio $\kappa$ turned out to be zero in this example (see fifth-order interleaver design example at the end of Sect. 9.3.1)

Another method is based on manipulating the phase response in one of the MZI branches in a periodic and nonlinear way, so that the phase change due to the length difference $\Delta L$ is approximately compensated near the passband and stopband centres (flattening the frequency response there), while the phase will change quickly near the band edges, sharpening the transfer function there. An example is shown in Fig. 9.10, where a ring resonator coupled to one of the branches acts as an allpass filter having a strongly nonlinear phase response with a frequency period equal to half the FSR of the MZI. Again, some fine-tuning facilities $\Delta \varphi_{k}$ are generally needed. An analysis is given in Sect. 9.3.2. Several architectures and design methods for interleavers incorporating both lattice-type structures and ring resonators or other feedback elements have been described in the literature, e. g. general design strategies and overviews [15-19], a generic design algorithm [20], and various more specific design topics [21-25]. 


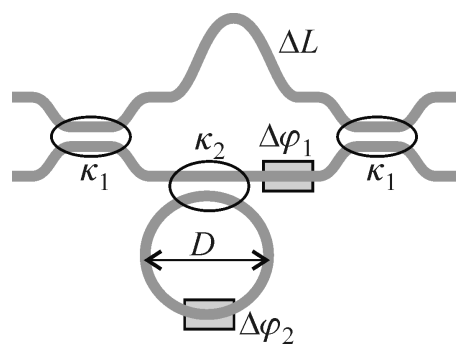

Fig. 9.10. Band flattening: MZI with internal phase response modified by a ring resonator

Signal transmission through an optical filter may be affected by dispersion, which tends to distort the pulse shape and hence may cause an increased bit error rate. In some cases it is possible to build an interleaver from two complementary sections having inverse phase responses, thus realising an overall response with negligible dispersion. A general discussion of dispersion of different interleaver types is given in [26]. The dispersion characteristics of both lattice-type and MZI + ring-type interleaving filters, as well as possible compensation techniques will be discussed in Sect. 9.4.

As indicated before, interleavers can be cascaded in order to build full (de)multiplexers and add-drop multiplexers. Here, the application of planar optical waveguide technology pays off, because it enables the integration of many stages into a single planar optical circuit. The different requirements for the consecutive stages will be discussed in Sect. 9.5 where the design and realisation of a tuneable 1 from 16 add-drop multiplexer will be explained as an example.

Finally, in Sect. 9.6, an overview will be given of technologies that have been used for fabricating interleaver-circuits, and examples will be given, including bulk optics, fibre-based implementations, and planar guided wave optics. Also, the characteristics of some commercially available interleavers, based on bulk optics, fibre optics, and integrated optics will be shown.

\subsection{Basic Mach-Zehnder Interferometer Interleavers}

The basic lay-out of a Mach-Zehnder interferometer- (MZI-) based interleaver is shown in Fig. 9.6b, and its power transfer function is given in Fig. 9.7. The MZI can be considered as a concatenation of three building blocks, the input directional coupler, a differential delay section, and the output coupler. Although the MZI is a relatively simple device that does not require a specific approach for analysis, we will use it for demonstrating 
a general approach that is applicable to a large class of periodic frequency response filters that would be difficult to analyse otherwise.

Following the approach of Madsen and Zhao [27], this section will start with a short summary of the main analytical tool for analysing filters that have a periodic frequency response, the Z-transform [28] and its relation with the traditional transfer function. The MZI is a four-port device, and so are the lattice-type filters that will be discussed in later sections. Therefore, a brief review will be given of the transfer matrix, describing a four-port network. Next, the optical properties of the basic building blocks of an MZI, waveguides and directional couplers, will be summarised. The properties of an MZI interleaver then follow from putting all the pieces together. Although a simple MZI does not have the most desirable interleaver properties, it serves well as a building block for composite interleaver filters, and the mathematical analysis of this relatively simple device is easily extended to the more interesting higher-order interleavers to be discussed in following sections.

\subsubsection{The Transfer Function and the Z-Transform}

The type of wavelength slicers to be analysed have a periodic transfer function in frequency, which is caused by the periodic phase response of a time delay. A lossless straight waveguide section of length $L$ has a transfer function

$$
H_{\text {straight }}=\mathrm{e}^{-\mathrm{i} k_{0} n_{\text {eff }} L}
$$

where $k_{0}=2 \pi f / c$. The propagation delay through the waveguide is $\tau=L n_{e f f} / c$, so that the transfer function (9.4), which just corresponds to a time delay, can be written explicitly as a function of frequency

$$
H_{\text {delay }}(f)=\mathrm{e}^{-\mathrm{i} 2 \pi f \tau}
$$

which clearly shows the frequency-periodic phase response. In an MZI there is only a single effective differential time delay $\tau$, corresponding to a frequency period $\Delta f=1 / \tau$. In the lattice filters and MZI + ring structures to be discussed later, there are multiple delays, which, however, are all integer multiples of an elementary delay $\tau_{0}$. It then makes sense to introduce a normalised frequency $f^{\prime}$

$$
f^{\prime}=f \tau_{0}
$$


which allows us to write (9.5) as

$$
H_{\text {delay' }}\left(f^{\prime}\right)=\mathrm{e}^{-\mathrm{i} 2 \pi f^{\prime}}
$$

\section{The Z-Transform}

If we now introduce a new complex variable $z$

$$
z=\mathrm{e}^{\mathrm{i} 2 \pi f^{\prime}}
$$

(9.7) can be written as

$$
H_{\text {delay }}(z)=z^{-1}
$$

The usefulness of this new variable is given by the property that the transfer function of $n$ times the unit delay is simply $z^{-n}$, which greatly simplifies the transfer functions of more complicated periodic filters, which can be expressed as rational functions in $z^{-1}$.

The mapping (9.8) of $f$ (or $f^{\prime}$ ) to the complex $z$-plane is known as the Ztransform, which is widely used in digital filter design. It can be considered as an analytic extension of the discrete-time Fourier transform (DTFT) for discrete signals [27]. The complex quantity $z$ takes values on the unit circle, starting at $z=1$ and making a full turn around the origin as $f^{\prime}$ varies from 0 to 1 .

From a given transfer function $H(z)$ in the $z$-domain - also known as the system function - the frequency response is found by evaluating $H(z)$ on the unit circle in the $z$-plane, i. e. $z=\mathrm{e}^{\mathrm{i} 2 \pi f^{\prime}}$.

The transfer function can be written as a ratio of $M^{\text {th }}$ - and $N^{\text {th }}$-order polynomials, or in an equivalent product form, e. g. [29], explicitly showing the zeros $z_{m}$ and poles $p_{n}$ of $H(z)$,

$$
H(z)=\frac{\sum_{m=0}^{M} b_{m} z^{-m}}{1+\sum_{n=1}^{N} b_{n} z^{-n}}=\Gamma z^{N-M} \frac{\prod_{m=1}^{M}\left(z-z_{m}\right)}{\prod_{n=1}^{N}\left(z-p_{n}\right)}
$$

where $\Gamma$ is the gain. The transfer function of a passive filter can never be greater than one, implying a maximum value of $\Gamma$ that is determined by $\max \left(|\mathrm{H}(\mathrm{z})|_{z=\mathrm{e} 2 \mathrm{pf}} \mathrm{f}^{\prime}\right)=1$. Since the real frequency transfer function is found for $z=\mathrm{e}^{\mathrm{i} 2 \pi f^{\prime}}$, only zeros that occur on the unit circle will correspond to zero transmission at the frequency corresponding to the argument of that zero.

A convenient graphical way to represent the transfer function is the pole-zero diagram. It shows the locations of each pole and zero in the 
complex plane. All zeros are designated by ' $\mathrm{o}$ ' and a pole is marked by ' $\mathrm{x}$ '. An example of a pole-zero diagram is depicted in Fig. 9.13 in Sect. 9.2.4.

A filter that has only zeros in its transfer function has only feed-forward paths and is classified as a finite impulse response (FIR) or moving average (MA) filter. Filters containing feed-back paths will have at least one pole in their transfer function, and are classified as infinite impulse response (IIR) filters. Sub-types of IIR filters contain either only poles (autoregressive (AR) filters), or both, poles and zeros (autoregressive moving average (ARMA) filters).

\section{The Frequency Response}

The frequency response function $H(f)$ is a complex function of $f$. It is usually expressed in terms of its magnitude $|H(f)|$ and phase $\varphi(f)$

$$
H(f)=|H(f)| \mathrm{e}^{\mathrm{i} \varphi(f)}
$$

Of course, the overall transfer function of a series connection of $M$ transfer functions $H_{i}$ is given by the product $\Pi H_{i}$, implying a multiplication of magnitude responses $\left|H_{i}(f)\right|$ and summation of phase responses $\varphi_{i}(f)$.

Since $H(f)$ is obtained by evaluating $H\left(z^{-1}\right)$ on the unit circle, the square of the magnitude response can be found as follows - if the coefficients of the transfer function are real [30]

$$
|H(f)|^{2}=H(f) H^{*}(f)=H(f) H(-f)=\left.H(z) H\left(z^{-1}\right)\right|_{z=\mathrm{e}^{\mathrm{i} 2 \pi f^{\prime}}}
$$

where $H^{\prime}$ denotes the complex conjugate of $H$. Equation (9.12) implies that reciprocal zeros, which are mirror images of each other about the unit circle, have identical magnitude characteristics. Based on the pole-zero representation (9.10) of $H(z)$, only the distance of each pole and zero from the unit circle, i. e. $\left|\mathrm{e}^{\mathrm{i} 2 \pi f^{\prime}}-p_{n}\right|$ or $\left|\mathrm{e}^{\mathrm{i} 2 \pi f^{\prime}}-z_{m}\right|$, affects the magnitude response. It can be shown that zeros $z_{m}, z_{m}^{*}, 1 / z_{m}$ and $1 / z_{m}^{*}$ all have the same magnitude response. However, their phase characteristics will be different, depending on $z_{m}$ being inside or outside the unit circle in the $z$-plane. A system with all zeros - and all poles as well - inside the unit circle $\left(\left|z_{m}\right|<1\right)$ has a so-called minimum-phase response (cf. Chap. 2) while all other systems (e.g. with one ore more zeros outside the unit circle) have a nonminimum phase response.

\section{Four-port Networks}

A basic $2 \times 2$ four-port device without reflection is schematically shown in Fig. 9.11. Its behaviour is conveniently described in terms of a transfer 


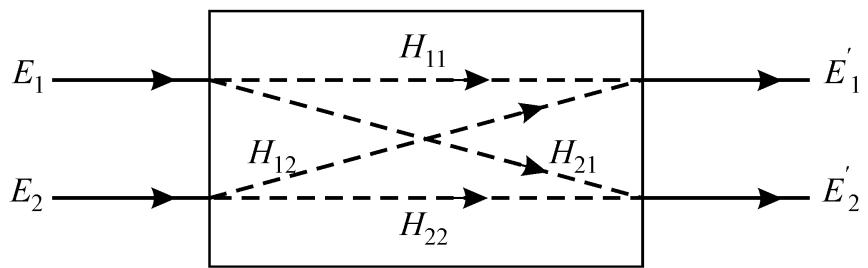

Fig. 9.11. Schematic drawing of the transfer functions in a $2 \times 2$ port without reflection

matrix relating the signals at its two output ports to those at the input ports, as given in (9.13),

$$
\left[\begin{array}{l}
E_{1}^{\prime} \\
E_{2}^{\prime}
\end{array}\right]=\mathbf{H}\left[\begin{array}{l}
E_{1} \\
E_{2}
\end{array}\right]=\left[\begin{array}{ll}
H_{11} & H_{12} \\
H_{21} & H_{22}
\end{array}\right]\left[\begin{array}{l}
E_{1} \\
E_{2}
\end{array}\right]
$$

where the complex transfer matrix $\mathbf{H}$ contains two so-called bar transfer functions $\left(H_{11}\right.$ and $\left.H_{22}\right)$ and two cross transfer functions $\left(H_{12}\right.$ and $\left.H_{21}\right)$.

If several such four-port devices, having transfer matrices $\mathbf{H}_{1}, \mathbf{H}_{2}, \ldots, \mathbf{H}_{n-1}$, $\mathbf{H}_{n}$ are concatenated, the transfer matrix $\mathbf{H}_{t o t}$ of the composite device is simply found by matrix multiplication, as

$$
\mathbf{H}_{t o t}=\mathbf{H}_{n} \mathbf{H}_{n-1} \cdots \mathbf{H}_{2} \mathbf{H}_{1}
$$

\subsubsection{Differential Delay Section}

The delay section of an MZI is formed by two independent waveguides having different lengths $L_{1}$ and $L_{2}$, respectively (we assume $L_{1}>L_{2}$ ). Although we assume almost identical branches (regarding phase constant $\beta(f)=k_{0} n_{\text {eff }}$ and attenuation coefficient $\alpha$ of the - single - guided mode), in order to model e. g. thermo-optic tuning, we do allow for a small deviation from the average effective index $n_{e f f}$, leading to an additional tuning phase delay $\varphi_{t}$ (indicated as $\Delta \varphi_{k}$ in Fig. 9.9) in branch 1 with respect to branch 2. The transfer matrix of the delay section is then given by

$$
\mathbf{H}_{\text {delay }}=\left[\begin{array}{cc}
\mathrm{e}^{-\alpha L_{1}} \mathrm{e}^{-\mathrm{i} k_{0} n_{e f f}(f) L_{1}} \mathrm{e}^{-\mathrm{i} \varphi_{t}} & 0 \\
0 & \mathrm{e}^{-\alpha L_{2}} \mathrm{e}^{-\mathrm{i} k_{0} n_{e f f}(f) L_{2}}
\end{array}\right]
$$

The differential delay $\tau$ is given as

$$
\tau=\frac{1}{\Delta f_{\mathrm{FSR}}}=\frac{\left(L_{1}-L_{2}\right) n_{g}}{c}=\frac{\Delta L n_{g}}{c}
$$


where $\Delta f_{\mathrm{FSR}}$ is the FSR, defined in (9.3), $\Delta L$ is the path length difference, and $n_{g}$ is the group index defined as

$$
n_{g}=n_{e f f}+f \frac{\partial n_{e f f}}{\partial f}
$$

Taking branch 2 as a reference, the transfer matrix can be written in terms of $\tau$,

$$
\mathbf{H}_{\text {delay }}=\eta_{L_{2}} \mathrm{e}^{-\mathrm{i} \beta(f) L_{2}}\left[\begin{array}{cc}
\eta_{\Delta L} \mathrm{e}^{-\mathrm{i} 2 \pi f \tau} \mathrm{e}^{-\mathrm{i} \varphi_{t}} & 0 \\
0 & 1
\end{array}\right]
$$

where $\eta_{L 2}=\mathrm{e}^{-\alpha L 2}$ is the loss along the path $L_{2}$ and $\eta_{\Delta L}=\mathrm{e}^{-\alpha \Delta L}$ is the differential loss due to the path length difference $\Delta L$. Applying the frequency normalisation with respect to the free spectral range as mentioned in the previous section, and applying the Z-transform, we arrive at

$$
\mathbf{H}_{\text {delay }}=\eta_{L_{2}} \mathrm{e}^{-\mathrm{i} \beta(f) L_{2}}\left[\begin{array}{cc}
\eta_{\Delta L} z^{-1} \mathrm{e}^{-\mathrm{i} \varphi_{t}} & 0 \\
0 & 1
\end{array}\right]
$$

In the remainder of this chapter we will omit the factor $\eta_{L_{2}} \mathrm{e}^{-\mathrm{i} \beta L_{2}}$, since it indicates just a constant loss and a linear phase. Furthermore, we introduce a new variable $Z$, defined by

$$
Z^{-1}=\eta_{\Delta L} \mathrm{e}^{-\mathrm{i} \varphi_{t}} z^{-1}
$$

so that $Z=z$ in the lossless case and without additional tuning phase shift. In general, for $f^{\prime}$ varying from 0 to $1, Z$ will take values on a circle centred in the complex plane, with a radius $\eta_{\Delta L} \leq 1$, and starting with an argument offset $\varphi_{t}$ at $f^{\prime}=0$. The simplified transfer matrix for a differential delay becomes

$$
\mathbf{H}_{\text {delay }} \cong\left[\begin{array}{cc}
Z^{-1} & 0 \\
0 & 1
\end{array}\right] \text {. }
$$

\subsubsection{Directional Coupler}

Couplers are among the most elementary building blocks in planar lightwave circuits, performing the functions of splitting and combining guided optical waves. (Another variant are fibre couplers, which are widely used in fibre optics.) 


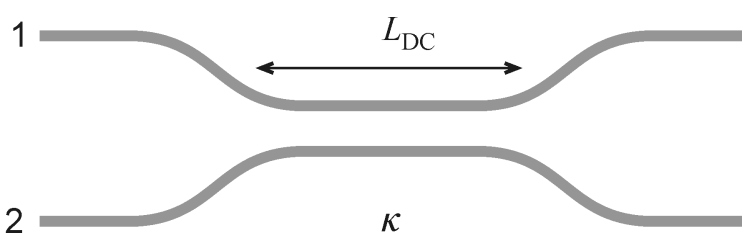

Fig. 9.12. Schematic top view of the directional coupler

Directional couplers (DC's) consist of two optical waveguides in close proximity to each other, see Fig. 9.12. Light will be coupled by the evanescent field of the mode, and power will be exchanged between the waveguides. The energy transfer process is similar to two mechanical pendulums that are weakly coupled by a spring. The two single-mode waveguides are usually chosen to be very close to each other in order to obtain a short coupling length.

One of the most convenient ways to understand the operation of a DC is by considering the two parallel waveguides as a single system, which supports two system modes. The modes of the input and output channels are decomposed into these system modes, and the power exchange between the separate waveguides can be simply described as an interference phenomenon of the system modes. It can easily be shown that the field amplitudes at the output channels, $E_{3}$ and $E_{4}$ are related to those at the input channels, $E_{1}$ and $E_{2}$ as

$$
\left[\begin{array}{l}
E_{3} \\
E_{4}
\end{array}\right]=\left[\begin{array}{cc}
\cos \psi & -\mathrm{i} \sin \psi \\
-\mathrm{i} \sin \psi & \cos \psi
\end{array}\right]\left[\begin{array}{c}
E_{1} \\
E_{2}
\end{array}\right]
$$

where the parameter $\psi$ is given as

$$
\psi=\frac{\pi f \Delta n_{e f f}}{c} L_{\mathrm{DC}}
$$

where $\Delta n_{\text {eff }}$ is the difference in effective index of the two system modes, and $L_{\mathrm{DC}}$ is the effective length of the coupling secton, as indicated in Fig. 9.12.

The coupling length $L_{\pi}$ is the propagation distance giving $\pi$ phase difference between the system modes

$$
L_{\pi}=\frac{c}{2 f \Delta n_{\text {eff }}}
$$

Arbitrary power splitting ratios can be obtained by simply choosing a length $L_{\mathrm{DC}}$ between 0 and $L_{\pi}$. The power is split equally over the output channels if the length of the coupler is half the coupling length. Equation 
(9.25) gives a shorthand notation for the transfer matrix of a DC (as defined in (9.22)),

$$
H_{\mathrm{DC}}=\left[\begin{array}{cc}
C & -\mathrm{i} S \\
-\mathrm{i} S & C
\end{array}\right]
$$

with

$$
\begin{gathered}
C=\cos (\psi)=\sqrt{1-\kappa} \\
-\mathrm{i} S=-\mathrm{i} \sin (\psi)=-\mathrm{i} \sqrt{\kappa}
\end{gathered}
$$

where $C$ is the bar transfer function, and $-\mathrm{i} S$ is the cross transfer function; $\psi$ is equal to the coupling strength integrated over the length and $\kappa$ is the power coupling ratio. Coupling does not only occur in the straight waveguides. It occurs already in the leads and the total phase is the sum of the phase in the straight waveguides and the leads.

\subsubsection{Mach-Zehnder Interferometer Interleaver}

The transfer matrix of the MZI can be calculated by simple multiplication of the transfer matrices of the type (9.19) and (9.25) of its component devices: input coupler, delay section, and output coupler.

$$
\mathbf{H}_{\mathrm{MZI}}=\mathbf{H}_{\mathrm{DC}_{2}} \mathbf{H}_{\text {delay }} \mathbf{H}_{\mathrm{DC}_{1}}
$$

Using the simplified delay transfer matrix (9.21), the MZI transfer matrix is found to be:

$$
\begin{aligned}
\mathbf{H}_{\mathrm{MZI}} & =\left[\begin{array}{ll}
H_{11}(z) & H_{12}(z) \\
H_{21}(z) & H_{22}(z)
\end{array}\right]=\left[\begin{array}{cc}
A(z) & B^{R}(z) \\
B(z) & A^{R}(z)
\end{array}\right]= \\
& =\left[\begin{array}{cc}
-S_{1} S_{2}+C_{1} C_{2} Z^{-1} & -\mathrm{i}\left(C_{1} S_{2}+S_{1} C_{2} Z^{-1}\right) \\
-\mathrm{i}\left(S_{1} C_{2}+C_{1} S_{2} Z^{-1}\right) & C_{1} C_{2}-S_{1} S_{2} Z^{-1}
\end{array}\right]
\end{aligned}
$$

The two polynomials in the left-hand column, called the forward polynomials, are labelled $A(z)$ for the bar transfer and $B(z)$ for the cross transfer, respectively. The two polynomials in the second column, called the reverse polynomials, are labelled $B^{R}(z)$ and $A^{R}(z)$, respectively. These reverse polynomials appear in the Z-transform description of many optical filters.

Note that the coefficients of the polynomial $A^{R}=H_{22}$ are in reverse order compared to those of $A=H_{11}$. The same holds for $B^{R}$ and $B\left(H_{12}\right.$ and $\left.H_{21}\right)$. 


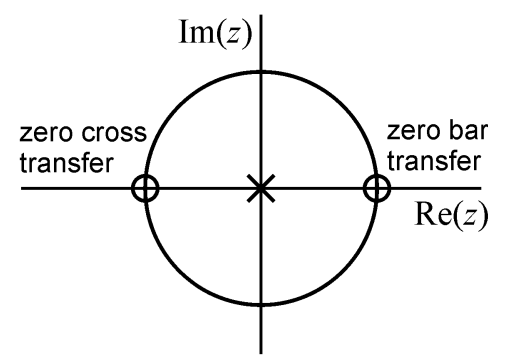

Fig. 9.13. Pole-zero diagram showing the zeros of the bar and cross transfer of the ideal MZI (lossless; perfect 3-dB couplers; no additional phase shift $\varphi_{t}$ )

This symmetry property allows calculating $H_{22}$ and $H_{21}$ if $H_{11}$ and $H_{12}$ are known. The transfer matrix can also be written in terms of the roots of the polynomials as follows:

$$
\mathbf{H}_{\mathrm{MZI}}=\left[\begin{array}{cc}
-S_{1} S_{2} Z^{-1}\left(Z-\frac{C_{1} C_{2}}{S_{1} S_{2}}\right) & -\mathrm{i} C_{1} S_{2} Z^{-1}\left(Z-\left(\frac{-S_{1} C_{2}}{C_{1} S_{2}}\right)\right) \\
-\mathrm{i} S_{1} C_{2} Z^{-1}\left(Z-\left(\frac{-C_{1} S_{2}}{S_{1} C_{2}}\right)\right) & -C_{1} C_{2} Z^{-1}\left(Z-\frac{S_{1} S_{2}}{C_{1} C_{2}}\right)
\end{array}\right]
$$

The bar transfer $A(z)$, for example, has a zero for $Z=C_{1} C_{2} /\left(S_{1} S_{2}\right)$, or, using (9.20), $z=\eta_{\Delta L} \mathrm{e}^{-\mathrm{i} \varphi_{t}} C_{1} C_{2} /\left(S_{1} S_{2}\right)$ and a pole at the origin $(z=0)$. A way to get insight into the polynomials is to plot all the poles and zeros in the complex $z$-plane, see Fig. 9.13. Their position in the $z$-plane depends on the coupling ratios and the tuning phase $\varphi_{t}$. The zeros always lie on the real axis when $\varphi_{t}=0$. The transfer is zero if $z$ is equal to a zero point and would be infinite if equal to a pole.

Since passive devices never have an infinite transfer, possible poles will never occur on the unit circle $z=\mathrm{e}^{\mathrm{i} 2 \pi f^{\prime}}$. An MZI transfer function, having a single pole at the centre, clearly satisfies this condition. The behaviour of a filter over its free spectral range can be investigated by evaluating its transfer matrix for all values of $z$ encountered by travelling once around the unit circle. The transfer goes to zero if $z$ crosses zero on the unit circle. However, a zero can also lie inside or outside the circle, see for example Fig. 9.17a. The closer $z$ (on the unit circle) gets to a zero the lower the transfer is. Two zeros at mirrored positions with respect to the unit circle $\left(z_{m}\right.$ and $\left.1 / z_{m}^{*}\right)$ will give the same amplitude transfer but a different phase transfer. The bar transfer $\left(H_{11}\right.$ and $\left.H_{22}\right)$ will have a zero on the unit circle, only if $\kappa_{1}=1-\kappa_{2}$, and the cross transfer $\left(H_{12}\right.$ and $\left.H_{21}\right)$ needs $\kappa_{1}=\kappa_{2}$ to have a zero on the unit circle. With $\kappa_{1}=\kappa_{2}=0.5$, both the bar and the cross 
transfer functions have a zero on the unit circle as shown in Fig. 9.13. When the two couplers are identical the matrix reduces to

$$
\begin{aligned}
\mathbf{H}_{\mathrm{MZI}} & =\left[\begin{array}{cc}
-S^{2}+C^{2} Z^{-1} & -\mathrm{i} S C\left(1+Z^{-1}\right) \\
-\mathrm{i} S C\left(1+Z^{-1}\right) & C^{2}-S^{2} Z^{-1}
\end{array}\right]= \\
& =\left[\begin{array}{ll}
-S^{2} Z^{-1}\left(Z-\frac{C^{2}}{S^{2}}\right) & -\mathrm{i} S C Z^{-1}(Z+1) \\
-\mathrm{i} S C Z^{-1}(Z+1) & -C^{2} Z^{-1}\left(Z-\frac{S^{2}}{C^{2}}\right)
\end{array}\right]
\end{aligned}
$$

This matrix shows that $H_{12}(z)$ and $H_{21}(z)$ will always have a zero on the unit circle, but $H_{11}(z)$ and $H_{22}(z)$ will also have a zero on the unit circle only, if $\kappa_{1}=\kappa_{2}=0.5$. This means that it is much easier to have complete isolation at the cross port than at the bar port.

For this case of two perfect $3 \mathrm{~dB}$ couplers where $C=S=\sqrt{1 / 2}$ (cf. (9.26)), the complete frequency response (optical power transfer) is found from (9.30) by substituting (9.20), (9.8) and (9.6) to be

$$
\left|H_{11}(f)\right|^{2}=\left|H_{22}(f)\right|^{2}=\eta_{\Delta L} \sin ^{2}\left(\frac{2 \pi f \tau}{2}+\frac{\varphi_{t}}{2}\right)+\frac{1}{4}\left(1-\eta_{\Delta L}\right)^{2}
$$

for the bar transfer and

$$
\left|H_{12}(f)\right|^{2}=\left|H_{21}(f)\right|^{2}=\eta_{\Delta L} \cos ^{2}\left(\frac{2 \pi f \tau}{2}+\frac{\varphi_{t}}{2}\right)+\frac{1}{4}\left(1-\eta_{\Delta L}\right)^{2}
$$

for the cross transfer. The lossless filter $\left(\eta_{\Delta L}=1\right)$ satisfies the simple condition $\left|H_{11}(z)\right|^{2}+\left|H_{21}(z)\right|^{2}=1$, which is obvious from power conservation.

Figure 9.14 shows the frequency response of the MZI filter for several values of the differential loss. Note that the filter curve has a very narrow stopband. The width of the stopband at $-25 \mathrm{~dB}$ is only $4 \%$ of the FSR.

The phase response of the bar transfer of the non-ideal MZI with identical directional couplers, from (9.30), is

$$
\varphi\left(f^{\prime}\right)=\tan ^{-1}\left(\frac{\frac{C^{2}}{S^{2}} \eta_{\Delta L} \sin \left(2 \pi f^{\prime}+\varphi_{t}\right)}{1-\frac{C^{2}}{S^{2}} \eta_{\Delta L} \cos \left(2 \pi f^{\prime}+\varphi_{t}\right)}\right)
$$

The non-linear frequency dependence of the phase response leads to a frequency-dependent group delay and dispersion as will be explained in Sect. 9.4. 


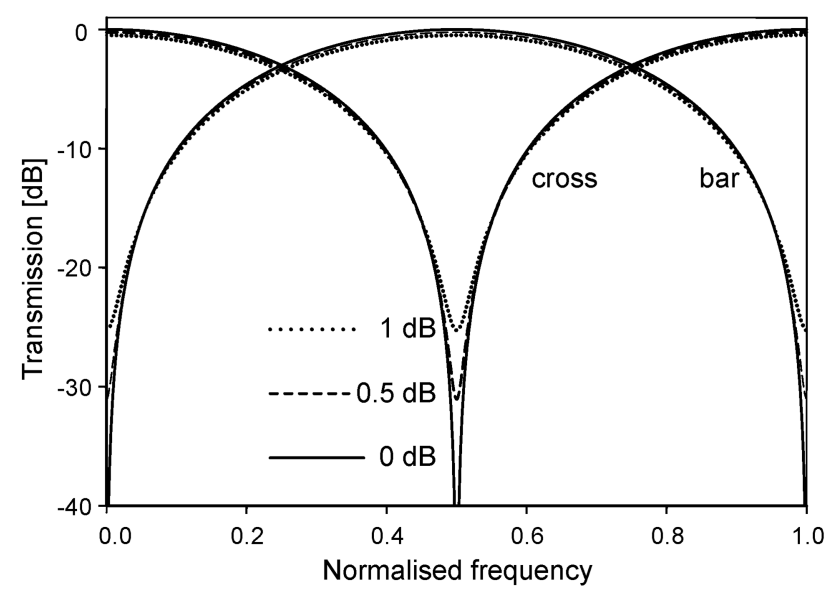

Fig. 9.14. Magnitude response for the Mach-Zehnder interferometer filter with differential loss of $0,0.5$, and $1 \mathrm{~dB}$, respectively; tuning phase $\varphi_{t}=0$, and coupling constants $\kappa_{1}=\kappa_{2}=0.5$

\subsection{Higher Order Interleavers}

\subsubsection{Finite Impulse Response Filters: The Resonant Coupler Approach}

The disadvantage of the MZI filter is that its transfer function is sineshaped. This results in a very narrow stopband. For example, if $25 \mathrm{~dB}$ isolation is required, the stopband width is only $8 \%$ of the channel spacing, so that $92 \%$ of the available spectrum must remain unused. Although the ideal rectangular-shaped filter transfer function, which would allow 100\% spectrum use, cannot be realized for reasons of causality, several approaches are known from the literature, e. g. [31], for improving the simple MZI filter. One of them involves resonant couplers (RC, also called multistage moving average filters or lattice filters) $[32,33]$. These filters can be implemented by cascading single MZI's, as shown in Fig. 9.15. Here a 2-stage filter is shown, consisting of 2 delay lines and 3 couplers. This concept can be extended to more stages. An $N$-stage filter has $N$ delay lines and $N+1$ couplers. The filter has 2 inputs and 2 outputs.

For simplicity the filters are assumed to have no loss. This means that the outputs are power complementary (the sum of the output powers is $100 \%$ ). The best way to design such a filter is by using the Z-transform description and the accompanying zero diagram as described above. One can find a synthesis algorithm in the literature, which calculates the power 


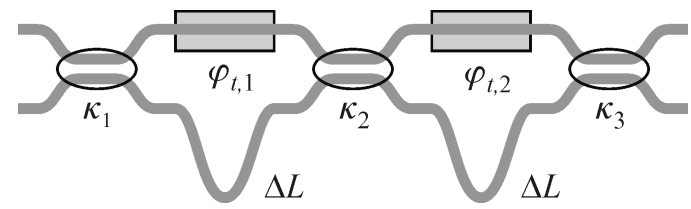

Fig. 9.15. Two stage resonant coupler filter, consisting of three couplers and two delay lines. Tuning phase shifts $\varphi_{t, 1}$ and $\varphi_{t, 2}$ might be provided by thermo-optic actuation

coupling ratios of each DC and the phase of the delay line from these polynomials [34, 27]. This is a very important algorithm, since it opens the way for using all the design tools for digital filters in order to design a desired filter that can then be mapped to a real optical filter layout. Due to chip space restrictions and optical losses, it is not possible to make an optical filter with a large number of delay lines. For example, a polynomial filter of order one hundred, which is very common in digital filters, is not (yet) possible. Also, every additional delay line needs an independent tuning element. Therefore it is important to design a filter using as few delay lines as possible.

\section{Filter Demands and Design Strategy}

In order to be useful as interleavers, for example cascaded in a binary tree arrangement, the filters must satisfy certain requirements, that can be summarised as follows:

- For interleaver operation, the bar transfer must be equal to the cross transfer shifted by half the FSR. This implies that the zero transfer frequencies are shifted by half the FSR compared to the frequencies of maximum transfer. The number of local maxima is equal to the number of local minima.

- To use the bandwidth as efficiently as possible, interleavers having broad passbands and stopbands are necessary.

- Low passband loss.

- Good isolation. It is difficult to fabricate filters having better isolation than $25 \mathrm{~dB}$.

Figure 9.16 summarises the filter synthesis process. There are four general steps in the process as described below. 


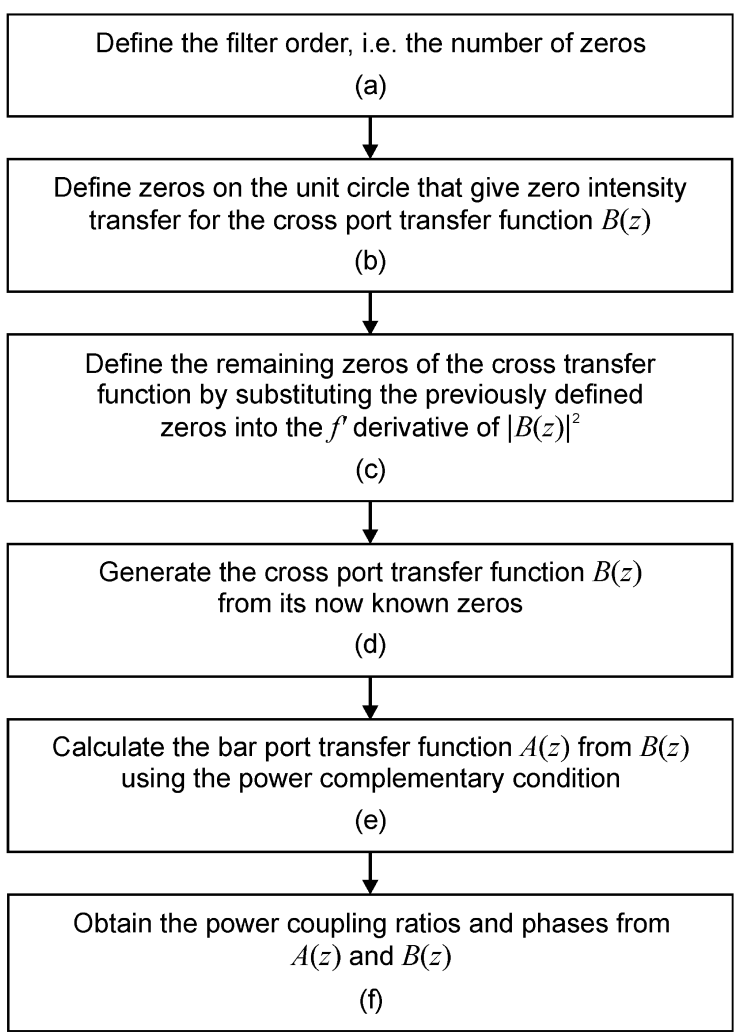

Fig. 9.16. Passband-flattened filter design synthesis flowchart

\section{(1) Definition of the filter order}

This first step, (a) in Fig. 9.16, defines the number of zeros, which is equal to the order of the filter. The maximum number of zeros will be limited by the fabrication accuracy and available space. Generally, the larger the order, the better a desired transfer function (e.g. a rectangular shape) can be approximated. The filter shape is adjusted by carefully locating the zeros in the complex $z$-plane. A zero placed on the unit circle will create a zero transfer (absolute minimum) and thus contributes to a stopband. Local maxima will arise between two adjacent zeros in the stopband. A zero inside or outside the unit circle will create a local minimum and, in the cases of practical interest, two surrounding local maxima. The overall effect can be a flattening of the passband if the zero is not positioned too close to the circle, so that the local minimum is shallow and only a small ripple remains. Due to the required symmetry of the passband and stopband of an interleaver, the number of passband maxima should equal the number of stopband zeros. This leads to the requirement of having one fewer zero in 
the passband than in the stopband, implying that the total filter order should be odd.

\section{(2) Generation of the cross port transfer function}

This step is represented by (b)-(d) of Fig. 9.16. To generate the cross transfer function, the zeros on the unit circle are positioned first. These zeros give zero intensity transfer at their normalised frequencies and thus define the stopband width. There are side-lobes between each pair of zeros in the stopband. Increasing the distance between the zeros leads to a broader stopband but also to a higher side-lobe level. Hence, for a given number of zeros on the unit circle, there is a trade-off between isolation and stopband width. A common requirement is to have the maximum side lobe level at $\leq-25 \mathrm{~dB}$.

Next the passband shaping zeros are positioned inside or outside the circle. These zeros will create local minima at their corresponding positions and two surrounding local maxima. They should be positioned so that the passband becomes a half FSR shifted mirror image of the stopband with respect to the $50 \%$ power transmission level.

Adopting the formalism with $A$ and $B$ polynomials as introduced in (9.28), and applying (9.12), the magnitude squared of a cross transfer function, $B(z)$, with real coefficients is

$$
|B(z)|^{2}=\left|B(z) B\left(z^{-1}\right)\right|_{z=\mathrm{e}^{\mathrm{i} 22 f^{\prime}}}
$$

while the transfer function can be written in terms of its roots as

$$
B(z)=\Gamma \prod_{m=1}^{M}\left(1-z_{m} z^{-1}\right)
$$

Substituting the unity zeros into (9.35) and applying (9.34), the magnitude squared function can be represented in terms of the unknown "passband" zeros and gain $\Gamma$. The locations of the maxima are given by the zeros of the derivative of (9.34) with respect to the normalised angular frequency $f^{\prime}$. Since these zeros of the derivative are independent of the unknown constant $\Gamma$, the locations of the "passband zeros" can be determined from (9.34) and (9.35)

$$
\frac{\mathrm{d}|B(z)|_{z=\mathrm{e}^{\mathrm{i} 2 \pi f^{\prime}}}^{2}}{\mathrm{~d} f^{\prime}}=\Gamma^{2} \prod_{m=1}^{M} \frac{\mathrm{d}}{\mathrm{d} f^{\prime}}\left[\left.\left(1-z_{m} z^{-1}\right)\left(1-z_{m} z\right)\right|_{z=\mathrm{e}^{\mathrm{i} 2 \pi f^{\prime}}}\right]=0
$$

or

$$
\prod_{m=1}^{M} \frac{\mathrm{d}}{\mathrm{d} f f^{\prime}}\left[\left.\left(1-z_{m} z^{-1}\right)\left(1-z_{m} z\right)\right|_{z=\mathrm{e}^{\mathrm{i} 2 \pi f^{\prime}}}\right]=0
$$


Finally, the transfer function (9.35) should be normalised to one, because the transmission of a passive device such as this Mach-Zehnder interferometer cannot exceed one. This procedure, which fixes $\Gamma$, is given by $(9.37)$,

$$
\max _{|z|=1}|B(z)|=\max _{\omega}|B(\omega)|=1
$$

\section{(3) Calculation of the bar port transfer function}

Once the cross transfer function has been obtained, the bar transfer function $A(z)$ can be calculated using the power complementary condition, (e) in Fig. 9.16. This condition, implying that the sum of the bar and the cross power transfer should be one, and assuming real coefficients of the transfer functions (allowing the use of (9.12)), provides a relationship between the $A$ and $B$ polynomials:

$$
|A(f)|^{2}=\left.A(z) A\left(z^{-1}\right)\right|_{z=\mathrm{e}^{\mathrm{i} 2 \pi f^{\prime}}}=1-\left.B(z) B\left(z^{-1}\right)\right|_{\mathrm{z}=\mathrm{e}^{\mathrm{i} 2 \pi f^{\prime}}}
$$

The bar transfer function $A(z)$ is obtained by calculating its $N$ zeros from (9.38). The $2 N$ zeros of $1-B(z) B\left(z^{-1}\right)$ appear as pairs of $\left(a_{k}, 1 / a_{k}^{*}\right)$ for $k=1 \cdot \cdot N$. Using spectral factorization, each zero of $A(z)$ is determined by selecting one from each pair of zeros of $1-B(z) B\left(z^{-1}\right)$. There are $2^{N}$ selections that can be made to obtain the zeros of $A(z)$. Thus, $2^{N}$ different versions of $A(z)$ can be obtained from one known $B(z)$. They have the same amplitude response but different phase characteristics.

\section{(4) Obtaining the optical parameters}

The last step, (f) in Fig. 9.16, is the generation of power coupling ratios and phases of each directional coupler and delay line of the filters from the bar and cross ports transfer functions. A simulation tool based on an algorithm derived by Jinguji and Kawachi [34] that can map the coefficients of the filter transfer function in a $z$ polynomial to the optical parameters is used. The algorithm uses recursion equations to calculate the power coupling ratios of each directional coupler and the phase of each delay line.

\section{Design Examples}

\section{Third order interleaver}

The first logical extension of the (first order) MZI would be a second order device with a second zero on the unit circle. As explained before, this extra zero can be positioned so as to effect stopband broadening. In order to also flatten the passband, an additional zero $\left(z_{3}\right)$ is needed, which is located 
symmetrically with respect to the first two zeros, but at the opposite side of the imaginary axis, i. e. on the real axis.

The zero diagram of this third-order filter is shown in Fig. 9.17a. The power transfer is shown in Fig. 9.17c. Here the distance between the two zeros $z_{1}$ and $z_{2}$ has been chosen so that the maximum of the side lobe is $-25 \mathrm{~dB}$. Increasing the distance results in a higher side lobe and broader stopband.

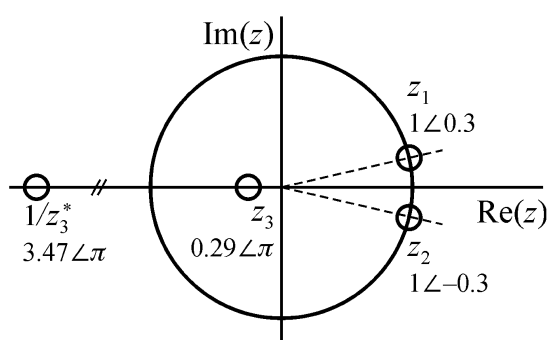

(a)

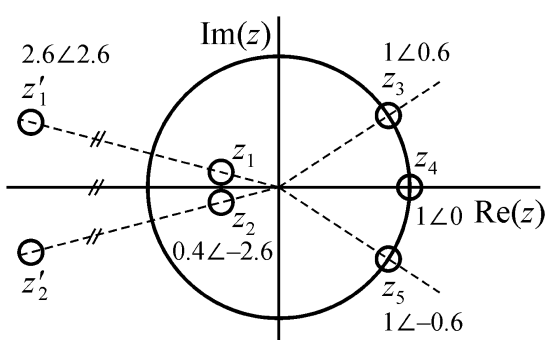

(b)

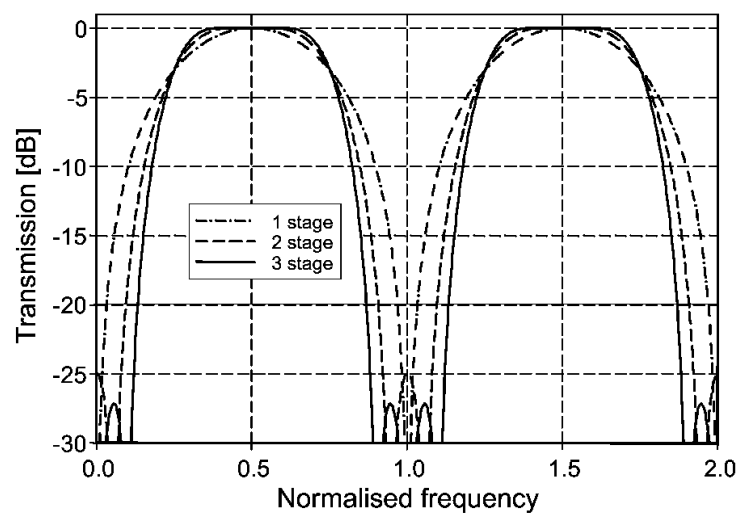

(c)

Fig. 9.17. (a) Zero diagram of the bar transfer $A(z)$ and the intensity transfer for the third order lattice filter. It has three zeros, two are on the unit circle $\left(z_{1}, z_{2}\right)$ and give a zero transfer; the third zero is on the real axis and can be chosen inside $\left(z_{3}\right)$ or outside $\left(1 / z_{3}^{*}\right)$ the unit circle. Both give the same amplitude transfer. (The notation $r \angle \varphi$ gives the modulus $r$ and the argument $\varphi$ [in radians] of complex $z$ ) (b) Zero diagram of the bar transfer $A(z)$ and intensity transfer for the fifth order lattice filter. It has five zeros, three are on the unit circle $\left(z_{3}, z_{4}, z_{5}\right)$ and give a zero transfer, two zeros are on the opposite side of the imaginary axis $\left(z_{1}, z_{2}\right)$ and can be chosen independently inside or outside the unit circle. Both choices give the same amplitude transfer. This graph must be mirrored about the origin to get the bar transfer. (c) Magnitude response for the 1, 2, and 3 stage slicer 
Proper slicer operation requires identical cross and bar amplitude transfer functions, shifted over half the FSR. This condition is satisfied by correctly positioning the third zero $z_{3}$. There is still a degree of freedom left, since the amplitude transfer does not change if $z_{3}$ is replaced by $1 / z_{3} *$. For both, the bar and the cross transfer, this zero can then be chosen to lie either inside (minimum phase) or outside (non-minimum phase) the unit circle, giving in total four possible solutions for this third order filter. These four different optical filter implementations have equal amplitude transfer but different phase transfer.

One of the couplers turns out to have $\kappa=0$, which means that this coupler is removed and the two neighbouring delay lines are combined into one having the double delay. The three-stage filter is reduced to one having two stages. Since the number of tuning elements is equal to the number of delay lines this implementation has also one tuning element less. The stopband width at $-25 \mathrm{~dB}$ is $14 \%$ of the FSR or $28 \%$ of the channel spacing; $72 \%$ of the band is unavailable for data transmission. Since the filter is power complementary, $-25 \mathrm{~dB}$ at the stopband can be calculated to correspond to a $0.014 \mathrm{~dB}$ ripple in the passband.

When looking at the four possible filter implementations, it can be seen that these four solutions can be split up into two groups where one contains mirror implementations of the other. This again shows that such a filter can be used both as filter and combiner. The last coupler can have a power coupling of either 0.923 or $0.077(=1-0.923)$. The implementation with power coupling 0.077 is preferable, since it is the shortest coupler, and therefore less sensitive to wavelength.

\section{Fifth order interleaver}

Further improvement of the filter curve can be obtained by adding two more zeros to the diagram, as shown in Fig. 9.17b. Three zeros are on the unit circle $\left(z_{3}, z_{4}, z_{5}\right)$ giving a broader stopband width (24\% of the FSR at $-25 \mathrm{~dB}$ ). The distance between these zeros is chosen so that the side lobes in the stopband are $-27 \mathrm{~dB}$. The other two zeros are placed at the opposite side of the imaginary axis and not on the unit circle to obtain passband flattening (three local maxima). Due to the lower stopband sidelobes, this filter is also better passband flattened, and of course the cross and bar transfer shapes are equal, but shifted by half the FSR. The two "passband zeros" can be chosen individually to be inside or outside the unit circle. This results in four possible configurations for these two zeros, giving sixteen solutions for the bar and the cross functions. Some of the solutions have one coupler with $\kappa=0$ allowing two neighbouring delay lines to be combined into a single one having the double delay. The best solution has two zero length couplers and chooses the shortest possible option for the remaining couplers. 


\subsubsection{An Infinite Impulse Response (IIR) Filter: MZI + Ring}

There is a different way to design a passband-flattened interleaver, by combining a ring resonator inside an asymmetric MZI [35-37] as shown in Fig. 9.18. More complex structures can be found in [38]. The ring introduces a frequency-dependent nonlinear phase shift in one arm, while maintaining a unity amplitude response. The frequency response of the ring will be discussed before going into the details of this interleaver.

Figure 9.19 shows the ring resonator and a waveguide coupled to that ring. Part of the light that propagates through the channel is coupled into the ring waveguide and travels through the ring. After one roundtrip, part of that light is coupled back into the straight waveguide and the remainder continues for a second roundtrip.

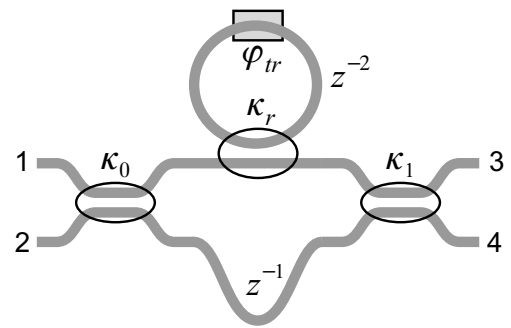

Fig. 9.18. Mach-Zehnder interferometer plus ring filter

This process continues until there is a stable solution. Two interrelated interference phenomena can be distinguished. First, there is the ring, where the total field distribution arises from waves that have made a number of roundtrips in the ring. Second, there is the straight waveguide where the direct light from the input interferes with waves that couple back from the ring. The output intensity is equal to the input if there is no loss. This interference clearly depends on the wavelength of the light. The roundtrip time of light propagating in the ring is $\tau_{r}$, but we allow a small deviation in the average effective index $n_{\text {eff }}$ of the ring, providing a phase adjustment $\varphi_{t r}$. The ring is in resonance, and intensity increases in the ring, if the total roundtrip phase $\varphi_{\text {ring }}=2 \pi f \tau_{r}+\varphi_{t r}=2 \pi \mathrm{m}$, where $m$ is an integer.

The light that couples back into the channel is in anti-phase with the light from the input and will interfere destructively. The ring is in antiresonance when $\varphi_{\text {ring }}=\pi(2 m+1)$. 
Introducing $z_{r}=\mathrm{e}^{\mathrm{i} 2 \pi f \tau_{r}}$, the transfer of the ring can be described by the following Z-transform polynomial [Ref. 27, pp. 306]

$$
H_{r}\left(z_{r}\right)=\frac{\mathrm{e}^{-\mathrm{i} \varphi_{t r}}\left(c_{r} \mathrm{e}^{+\mathrm{i} \varphi_{t r}}-\eta_{r} z_{r}^{-1}\right)}{1-c_{r} \mathrm{e}^{-\mathrm{i} \varphi_{r r}} \eta_{r} z_{r}^{-1}}=\frac{c_{r}\left(z_{r}-\frac{\eta_{r}}{c_{r}} \mathrm{e}^{-\mathrm{i} \varphi_{t r}}\right)}{z_{r}-\eta_{r} c_{r} \mathrm{e}^{-\mathrm{i} \varphi_{t r}}}
$$

where $c_{r}=\sqrt{1-\kappa_{r}}, \eta_{r}=\mathrm{e}^{-2 \pi \alpha_{r} r}, \alpha_{r}$ is the ring waveguide attenuation coefficient and $r$ is the ring radius.

We consider the lossless case, where the ring resonator acts as an allpass filter producing a non-linearly frequency-dependent phase shift, which is given by

$$
\varphi_{r}(f)=\tan ^{-1}\left[\frac{\left(1-c_{r}^{2}\right) \sin \left(2 \pi f \tau_{r}+\varphi_{t r}\right)}{2 c_{r}-\left(1+c_{r}^{2}\right) \cos \left(2 \pi f \tau_{r}+\varphi_{t r}\right)}\right]
$$

and is shown in Fig. 9.19b for different values of the modulus of the pole location, $\left|z_{p}\right|$. In this lossless case $\left|z_{p}\right|=c_{r}$ is found from (9.39). The extreme case $\left|z_{p}\right|=c_{r}=0$ corresponds to a power coupling constant $\kappa=1$, meaning that all the light couples from the input into the ring, makes exactly one roundtrip, and then couples back completely to the straight waveguide. This is equivalent to a single waveguide, which is lengthened by an amount equal to the circumference of the ring. As expected, its phase response is linear. For $\left|z_{p}\right|=0.9$ only a small part of the power is coupled into the ring.

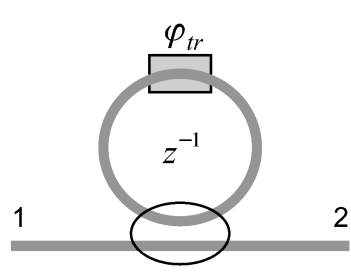

$\kappa_{r}$

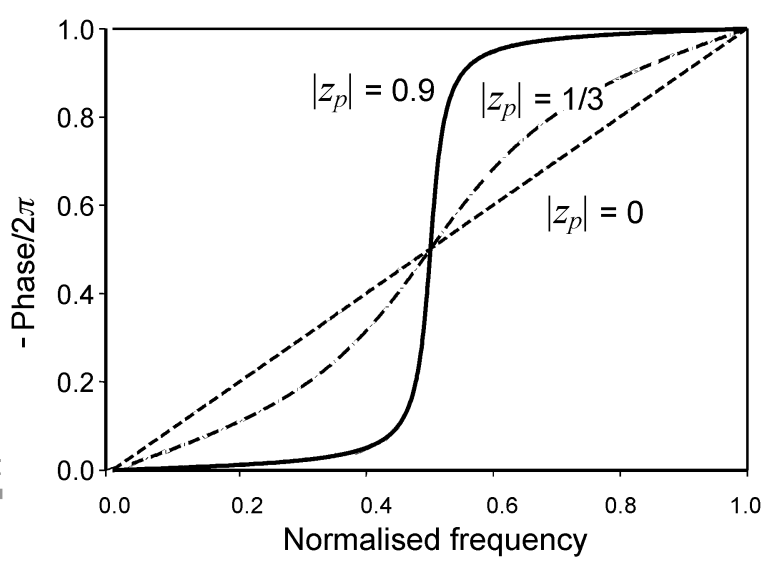

(b)

Fig. 9.19. (a) $1 \times 1$ port ring filter; (b) phase response for a lossless ring resonator with three different pole locations $\left|z_{p}\right|$, assuming that $\varphi_{t r}=\pi$ 
Near resonance a high intensity builds up and the phase changes rapidly in a nonlinear fashion.

This non-linear phase shift can now be used inside the MZI (Fig. 9.18), where the ring is connected to the short channel. The time delay (or roundtrip length) of the ring should be exactly twice the differential time delay of the MZI $\left(\tau_{t}\right)$, since the periodic nonlinear phase change should occur synchronously with the periodic MZI response curve in order for the ring to effect passband flattening and stopband broadening. The intensity transfer from port 1 to 3 (bar) and from 1 to 4 (cross) for the MZI + ring is given by (9.41) and (9.42) respectively,

$$
\begin{aligned}
\left|H_{11}(f)\right|^{2} & =\sin ^{2}\left(\frac{\Delta \varphi(f)}{2}\right) \\
\left|H_{12}(f)\right|^{2} & =\cos ^{2}\left(\frac{\Delta \varphi(f)}{2}\right)
\end{aligned}
$$

where the DC's of the MZI have both $\kappa=0.5$, and $\Delta \varphi(f)=\varphi_{r}(f)-2 \pi f \tau_{t}$, the difference between the phase of the ring-path and the phase of the through-path arm of the MZI. The bar transfer $\left|H_{11}(f)\right|$ is zero when $\Delta \varphi(f)=2 m \pi$, where $m$ is an integer; it is one for $\Delta \varphi(f)=(2 m+1) \pi$. Passband flattening can be obtained by tuning the ring to be in anti-phase $\left(\varphi_{t r}=\pi\right)$ at maximum transfer of the MZI.

Figure 9.20 shows the result obtained by adding the ring, with $\varphi_{\mathrm{tr}}=\pi$. The intensity transfer is passband flattened and stopband broadened. The second graph shows the frequency-dependent phase of the two arms of the MZI with respect to the short arm of the same MZI without ring. The alternating long-short-dashed line represents the phase of the long arm (the one without ring). It has a phase change of $2 \pi$ in one FSR. The dashed line gives the phase of the short branch in the case of $100 \%$ coupling to the connected ring. It has a phase change of $4 \pi$ in one period $\left(\tau_{r}=2 \tau\right)$. The solid line shows the phase for $\kappa_{r}=0.82$ power coupling to the ring. The phase oscillates around the dashed line. There are exactly two periods of oscillations. The intensity transfer of the filter is now determined by the phase difference between the two channels as shown in the last graph. The centres of the passband and stopband occur at a phase difference of $m \pi$. The coupling coefficient has been calculated in such a way that near the centre of the pass- and stopbands the phase slope of the short branch + ring is equal to that of the long branch, resulting in a constant zero (or $\pi$ ) phase difference between the branches over a large fraction of these bands. As a result there is almost no change in the transfer. It is important that this stability 
occurs at a maximal or minimal transfer, which is obtained by careful tuning of the phase of the ring relative to the MZI. The local maxima in the stopband occur at the frequency where the slope of the phase difference is zero. There is a rapid transition in the transfer from passband to stopband, because the ring is in resonance, which results in a fast phase change.

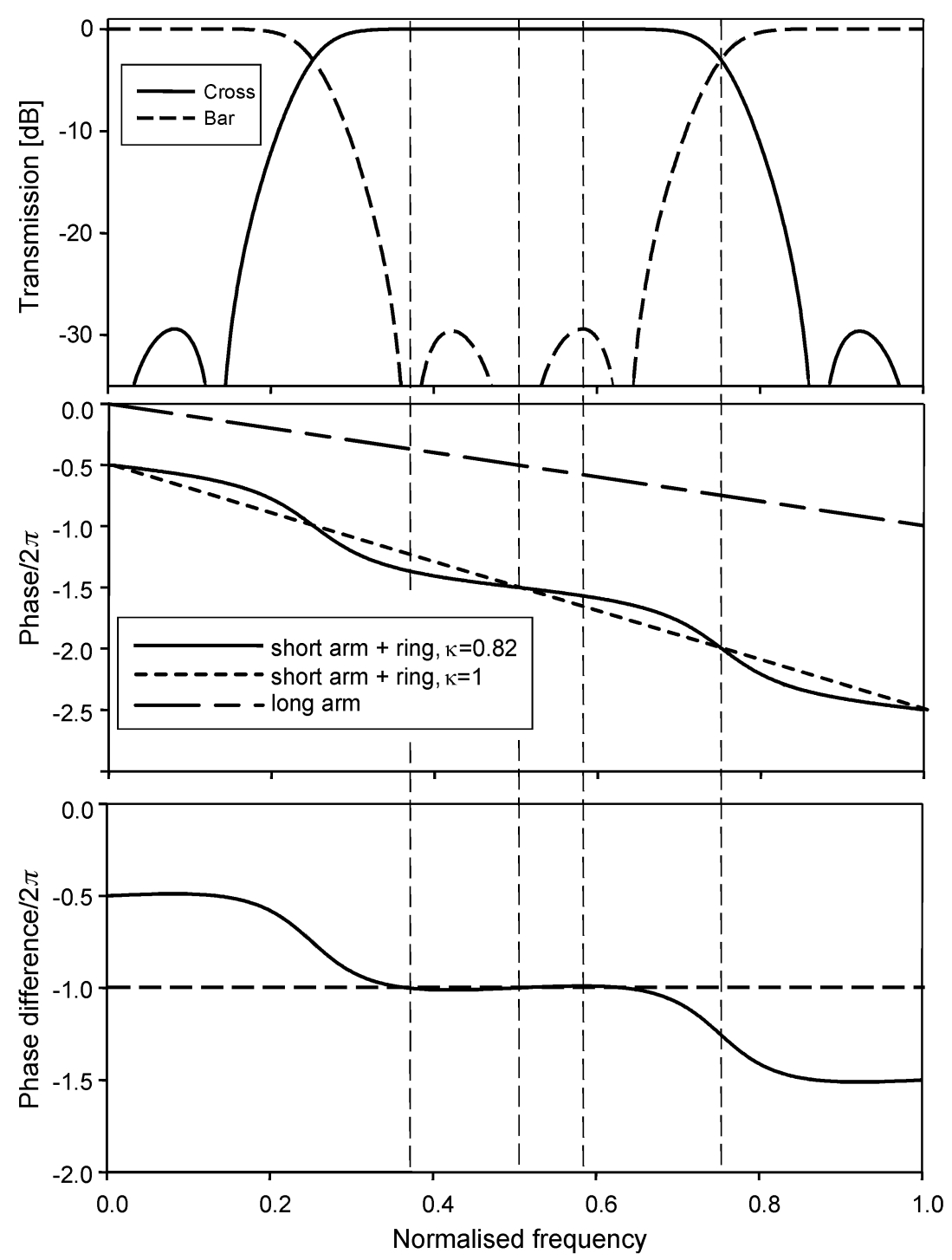

Fig. 9.20. Intensity transfer, phase of each arm (with respect to the short arm without ring), and the phase difference of a lossless correctly tuned MZI + ring filter with perfect $3 \mathrm{~dB}$ couplers, and $\varphi_{\mathrm{tr}}=\pi$ 


\subsection{Group Delay and Dispersion}

Dispersion is a measure of linearity of the phase response with respect to the frequency. The group delay is the local slope of the phase response curve, i. e., the slope of the phase at the frequency being evaluated. A filter's group delay or envelope delay is defined as the negative derivative of the phase response with respect to angular frequency $\omega$ as follows [30]:

$$
\tau_{g}(\omega)=-\frac{\mathrm{d} \varphi(\omega)}{\mathrm{d} \omega}
$$

If $\varphi$ is in radians and $\omega$ is in radians per second, then the absolute group delay is given in seconds. For a sequence of discrete signals, each stage has a delay that is an integer multiple of a unit delay, $\tau_{0}$. If the angular frequency is normalised to $\tau_{0}$ such that $\omega^{\prime}=\omega \tau_{0}$, then the normalised group delay $\tau_{\mathrm{g}}^{\prime}$ is given in number of unit delays $\tau_{0}$, leading [27] to:

$$
\tau_{g}=\tau_{0} \tau_{g}^{\prime}
$$

with a normalised group delay

$$
\tau_{g}^{\prime}\left(\omega^{\prime}\right)=-\frac{\mathrm{d} \varphi\left(\omega^{\prime}\right)}{\mathrm{d} \omega^{\prime}}=-\left.\frac{\mathrm{d}}{\mathrm{d} \omega^{\prime}} \arg (H(z))\right|_{z=\mathrm{e}^{\mathrm{i} \omega^{\prime}}}
$$

The filter dispersion is the derivative of the group delay. For normalised frequency $f^{\prime}=f \tau$, the normalised dispersion $D^{\prime}$ is [27]

$$
D^{\prime} \equiv \frac{\mathrm{d} \tau_{g}^{\prime}}{\mathrm{d} f^{\prime}}=2 \pi \frac{\mathrm{d} \tau_{g}^{\prime}}{\mathrm{d} \omega^{\prime}}
$$

and the filter dispersion $D$ in absolute units $[\mathrm{s} / \mathrm{m}]$ is [27]

$$
D=\frac{\mathrm{d} \tau_{g}}{\mathrm{~d} \lambda}=-c\left(\frac{\tau_{0}}{\lambda}\right)^{2} D^{\prime}
$$

In comparison, for optical fibres the dispersion $D_{f}$ is typically defined as the derivative of the group delay with respect to wavelength $\lambda$ and normalised with respect to length $L$ [27]

$$
D_{f}=\frac{1}{L} \frac{\mathrm{d} \tau_{g}}{\mathrm{~d} \lambda}
$$

Practical units for $D_{f}$ are $[\mathrm{ps} /(\mathrm{nm} \cdot \mathrm{km})]$. 


\subsubsection{MZI Group Delay and Dispersion}

The normalised group delay of the non-ideal MZI with identical directional couplers can be calculated from (9.33) and (9.45)

$$
\tau_{g}^{\prime}\left(\omega^{\prime}\right)=\frac{\frac{C^{2}}{S^{2}}\left[\frac{C^{2}}{S^{2}}-\cos \left(\omega^{\prime}\right)\right]}{1-2 \frac{C^{2}}{S^{2}} \cos \left(\omega^{\prime}\right)+\frac{C^{4}}{S^{4}}}
$$

Figure 9.21a shows the normalised group delay of the MZI for different coupling constants. Note that the ideal MZI $(\kappa=0.5 \rightarrow C=S)$ has a constant group delay and thus no dispersion. Figure $9.21 \mathrm{~b}$ shows the normalised dispersion of the MZI. Note that the dispersion sweep is in the stopband region and that dispersion is low in the passband region.

The group delay and dispersion go to infinity, as $\kappa$ goes to 0.5 . This is possible since this is in the stopband region and the intensity transfer goes to zero.

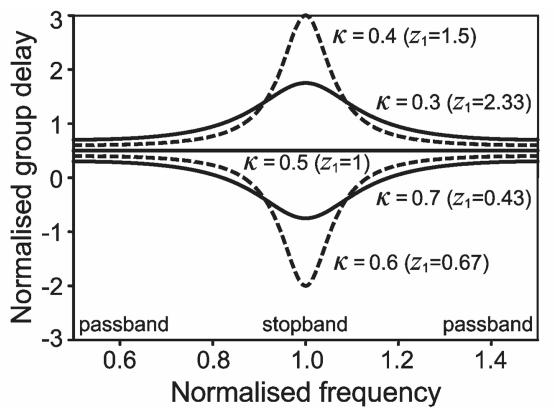

(a)

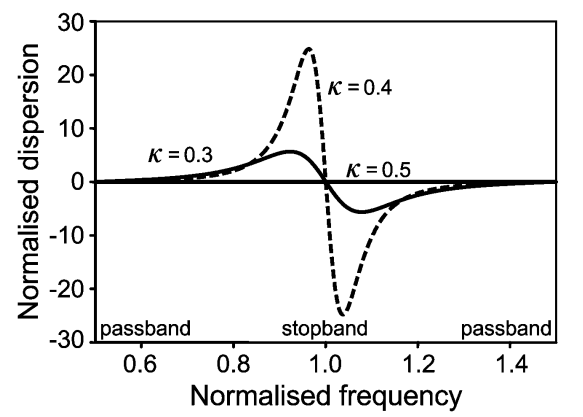

(b)

Fig. 9.21. Bar transmission of the MZI for various coupling constants. (a) Group delay. (b) Dispersion

\subsubsection{Third- and Fifth-Order Lattice Filter Group Delay and Dispersion}

The dispersion for the $3^{\text {rd }}$ order interleaver is non-zero as shown in Fig. 9.22a, in contrast to the ideal MZI. There is a frequency-dependent group delay having a minimum at the centre of the passband, resulting in a zero of the dispersion. The minimum normalised dispersion is -2.6 , which is equivalent to $1.9 \mathrm{~km}$ of standard single mode fibre for a $100 \mathrm{GHz}$ FSR filter. For the $5^{\text {th }}$ order interleaver, Fig. $9.22 \mathrm{~b}$, the dispersion goes to 

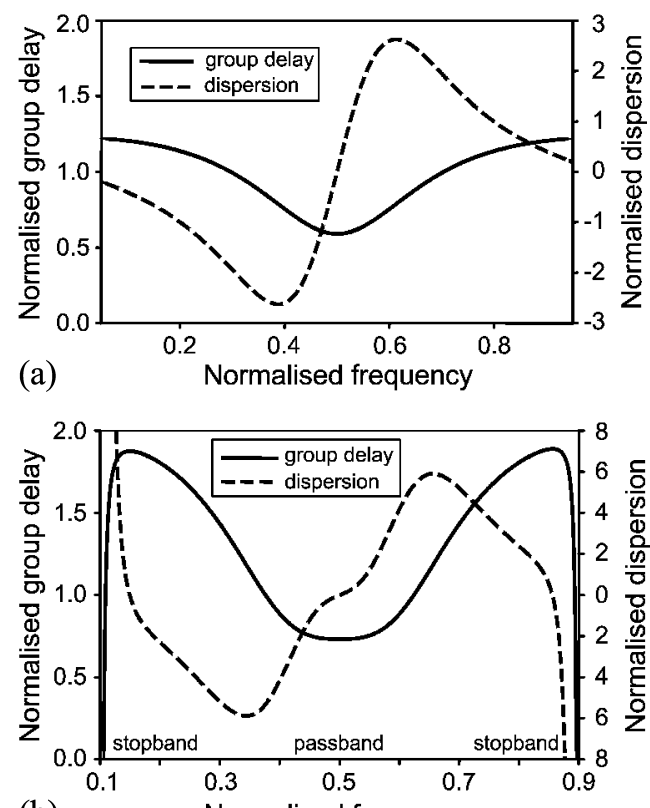

(b)

Normalised frequency

Fig. 9.22. Normalised group delay and normalised dispersion of bar response of minimum phase $3^{\text {rd }}$ and $5^{\text {th }}$ order filters. (a) $3^{\text {rd }}$ order 2-stage filter; the minimum and maximum dispersion are -2.6 and 2.6 , respectively. (b) $5^{\text {th }}$ order 3 -stage interleaver; the minimum and maximum dispersion are -5.9 and 5.9 , respectively

infinity when reaching the stopband, which is not interesting since the intensity is low. The minimum normalised dispersion is -5.9 , which is equivalent to $4.3 \mathrm{~km}$ of standard single mode fibre for a $100 \mathrm{GHz}$ FSR filter.

\subsubsection{MZI + Ring Group Delay and Dispersion}

Figure 9.23 shows the overall phase, delay, and dispersion of the bar transfer $H_{11}(z)$ of the MZI + ring filter. The normalised dispersion is zero at the centre of the passband and goes from negative to positive in the passband region. The extremes are -22 and +22 at a normalised frequency of 0.29 and 0.71 , respectively. The transfer is $-0.5 \mathrm{~dB}$ at these points. The dispersion of the filter does not depend on the chosen input and output ports. It will always give the same dispersion curve. So the dispersion will always be doubled when two MZI + ring interleavers are cascaded. 


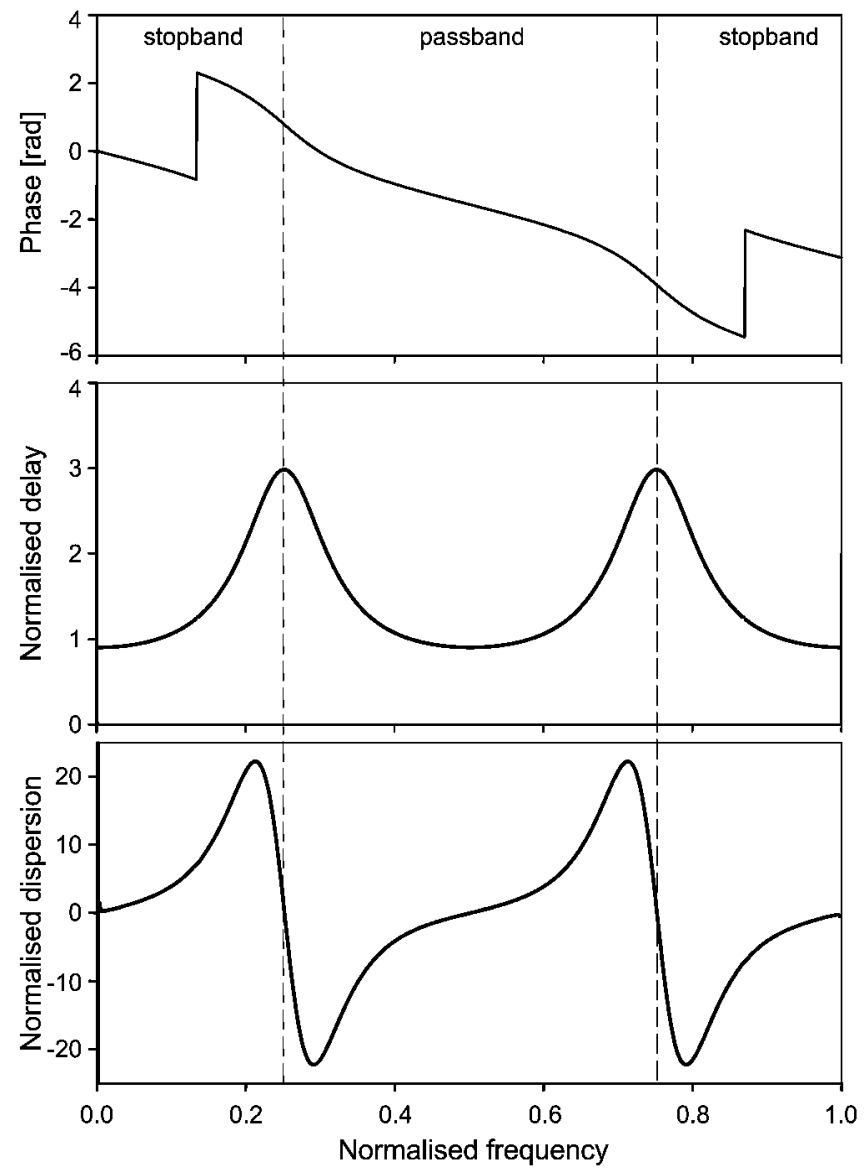

Fig. 9.23. Phase, normalised delay, and normalised dispersion of the cross transfer of MZI + ring filter

\subsection{Cascaded Interleavers}

As indicated in the introduction, interleavers can be used as building blocks to form more complicated optical functions. One way of cascading interleavers has been extensively discussed in Sect. 9.3.1. In this lattice architecture, the overall cascade had the same basic functionality as a single interleaver, but its transfer function was strongly improved.

One way of extending the functionality is to build a cascade based on a binary tree architecture as shown in Fig. 9.4. Full demultiplexing of $N$ channels requires $\mathrm{N}-1$ slicers. Therefore, optical integration technology is essential for economic fabrication of such composite devices, as pioneered 
by Verbeek et al. [4] and further developed by NTT, e. g. [39]. Compared to other multiplexers, for example the arrayed waveguide grating (AWG, see Chap. 4), this architecture does not seem to scale very well with the number of multiplexed optical channels. However, it offers a large degree of flexibility for engineering the transfer function, e. g. [23, 24], and especially for (fine-)tuning to individual channels and reconfiguring the assignment of optical signal channels to the output ports. The signals at the two output ports of an MZI interleaver are swapped by introducing a $\pi$ phase shift in one of the MZI branches. Since this can be done for each of the slicers in the binary tree, each wavelength channel can be routed to each of the output ports, and many - but by no means all - channel permutations can thus be obtained. In this way, a binary tree slicer arrangement combines the functionality of optical frequency (de)multiplexing with switching, which may make it an efficient architecture for a number of wavelength routing applications.

If full demultiplexing is not required, for example for dropping a single channel from a multiplexed signal, a partial binary tree may be used. Only $\log _{2} N$ slicers are required if the unwanted channels can be discarded. If the other channels should be kept, the partially demultiplexed signal must be remultiplexed using another identical partial binary tree. An example of a 1 from 8 add-drop multiplexer using this principle is shown in Fig. 9.5. The add-function comes "for free" with the drop function if a $2 \times 2$ interleaver is applied for the deepest stage, and a total of $2 \log _{2} N-1$ slicers is needed.

It is worth noting that the "quality" of the transfer function is not necessarily equal for each of the slicers in a cascade. The most critical stage is the first one, which has the smallest free spectral range (FSR), since its function is to separate adjacent channels. Its properties mainly determine the flatness and width of the passband and the adjacent channel isolation of the composite device. A lower order lattice filter can be used for the following stages, since the channel width will be a smaller fraction of the FSR of those stages. The principle is shown in Fig. 9.24.

The isolation of the drop channel with respect to the add channel deserves special attention, because these channels - separated by only a single large-FSR slicer in the configuration of Fig. 9.5 - have the same nominal frequency, and the level of the locally generated add signal may be relatively high. This may cause strong interference products within the bandwidth of the detection system (in-band crosstalk). The potential problem arising from this is elegantly solved by adopting an add-after-drop configuration at the expense of a single additional slicer, one of the " $D$ " blocks, as illustrated in Fig. 9.25. 


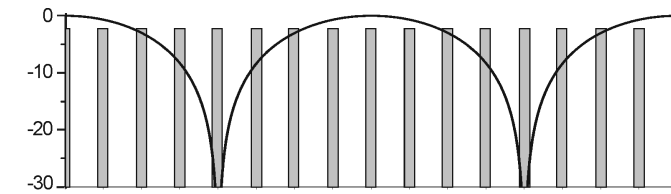

(a)

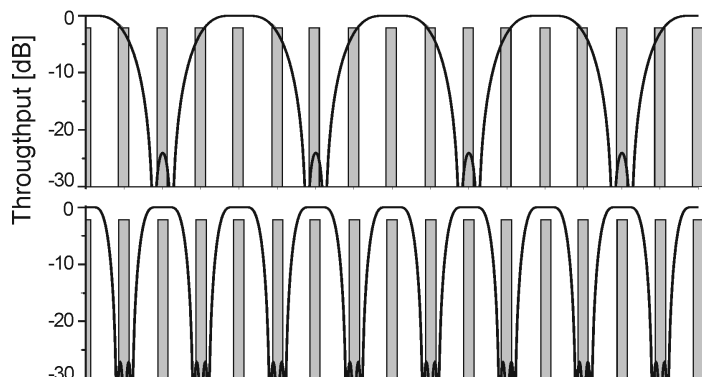

(b)

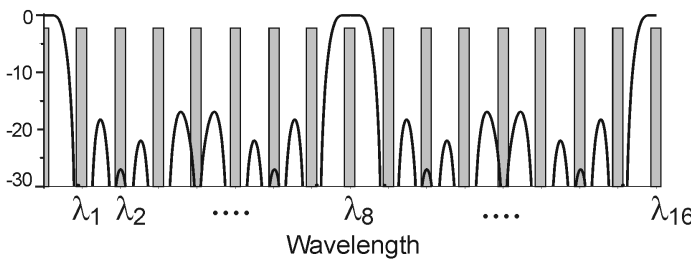

(c)

(d)

Fig. 9.24. Transfer functions of different lattice filters. (a) Single-stage MZI. (b) Twostage (third order). (c) Three-stage (fifth order). The ratios of the free spectral ranges of the slicers are 4:2:1 for slicers a, b, and c, respectively. (d) Transfer function from the input to one of the outputs of the binary tree cascade of these three interleavers

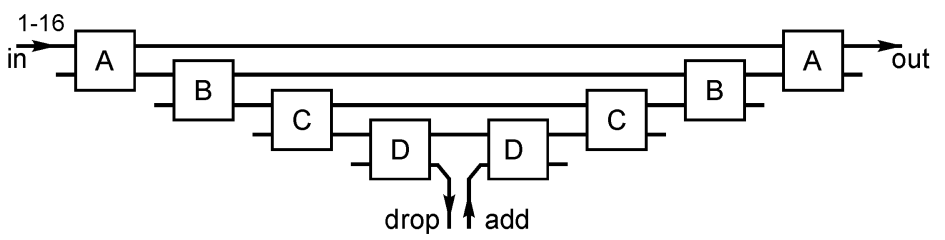

Fig. 9.25. Schematic drawing of 1 from 16 add-drop multiplexer with add-after-drop functionality for a $200 \mathrm{GHz}$ channel spacing. FSR ratios are 1:2:4:8 for A:B:C:D-type slicers respectively. For obtaining a $25 \mathrm{~dB}$ isolation over $>85 \mathrm{GHz}$ stopband, A-type slicers were chosen to have 3 stages, B-type 2 stages, and C and D-types 1 stage [5]

An example of the lay-out of a thermo-optically tuneable 1 from 16 addafter-drop multiplexer, based on partial binary trees of different order lattice filters, and designed to select channels on a $200 \mathrm{GHz}$ ITU grid in the C-band, is given in Fig. 9.26. The device was fabricated using silicon oxynitride technology $[40,41]$. Its in-to-drop and in-to-out characteristics when tuned to a given channel are shown in Fig. 9.27. 


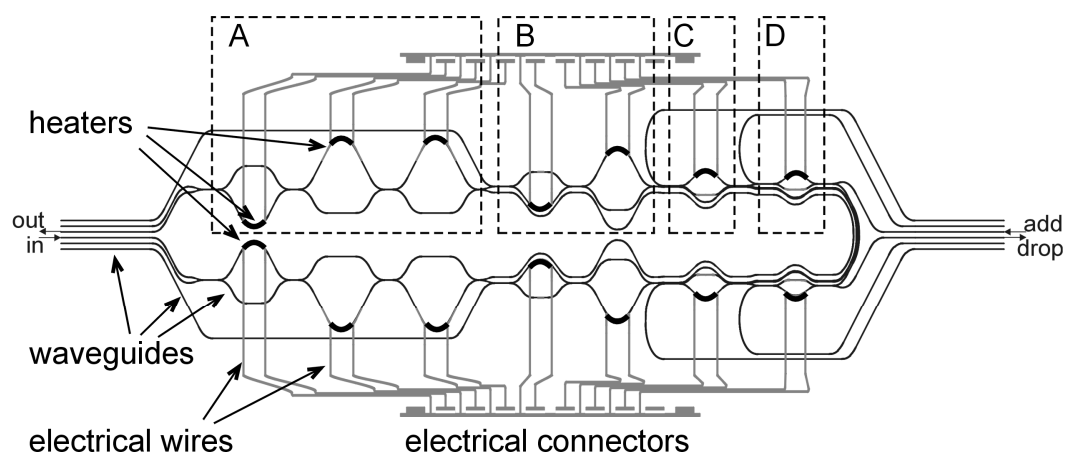

Fig. 9.26. Lay-out of a tuneable 1 from 16 add-drop multiplexer using the architecture shown in Fig. 9.25 [5]. The optical circuit has been folded about the vertical centre line. The locations of A, B, C, and D type slicers are indicated. Metal connections of the heaters used for thermo-optically tuning the individual delay sections are also shown

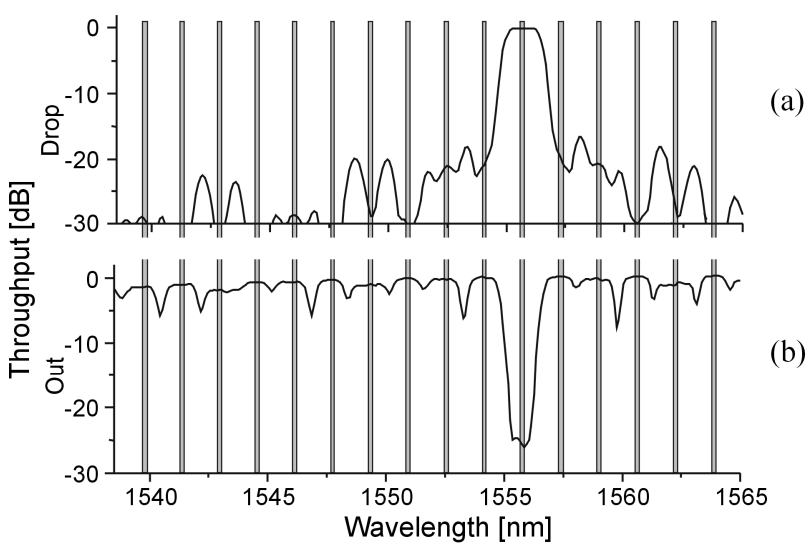

Fig. 9.27. Measured transfer functions: in-to-drop (a) and in-to-out (b) of the multiplexer shown in Fig. 9.26. The grey vertical bars indicate channels of the $200 \mathrm{GHz}$ ITU grid for which the filter was designed. Measurements were done with unpolarised light

In Sect. 9.3.2 the combination of an MZI with a ring resonator has been discussed. A different kind of such a combination, placing the ring outside the MZI (in fact just cascading ring and MZI) has been proposed by Vázquez et al. [42]. They introduce gain into the ring, leading to a strongly peaked transmission spectrum instead of the allpass characteristic described in Sect. 9.3.2. This might make the applicability of their configuration as an interleaver or demultiplexer in WDM systems questionable. 


\subsection{Realisations of Interleavers in various Fabrication Technologies}

As stated in the introduction, interleavers can be built from any type of wavelength filter having a periodic frequency response. This section aims at giving a flavour of recent realisations of interleavers based on different operating principles and using different technologies.

\subsubsection{Bulk and Fibre Optics}

Although this chapter focuses on integrated optical realisations of interleavers, most commercially available devices at the time of writing are based on bulk optics.

\section{Lattice Filters}

The implementations, based on birefringent crystals, that are equivalent to MZI lattice filters have been known for a long time as Lyot [43] or Solc [44] filters. Many different variations of the basic principle have been realised [1]. In such filters (see Fig. 9.28), which found their early applications mainly in astronomic instruments, the two fundamental polarisations in the crystals, the ordinary (o) and extraordinary (e) waves, experience a different refractive index $\left(n_{\mathrm{e}}-n_{\mathrm{o}}=\Delta n\right)$, and hence a frequency-dependent phase difference $\Delta \varphi$ given by

$$
\Delta \varphi=\frac{2 \pi}{c} f \Delta n L
$$

where $L$ is the length of the crystal. Equation (9.50) is very similar to (9.2). The angular offset between the optic axes of consecutive crystals causes a mixing of the o- and e-waves from one crystal to the next, just as the directional couplers do between consecutive delay sections in an MZI lattice filter. Instead of rotating the crystals, half-waveplates may be located in between the aligned crystals. The device of Fig. 9.28 works only with a single input polarisation, but, exploiting the complementary mixing properties of orthogonal polarisations in a configuration as shown in Fig. 9.29, polarisation-independent operation can be obtained [45].

Instead of birefringent crystals, also artificial birefringent units can be used, consisting of polarisation beam splitters providing two different propagation paths (through different isotropic materials, e. g. glass blocks) for the two orthogonal polarisations, as illustrated in Fig. 9.30 [46]. 


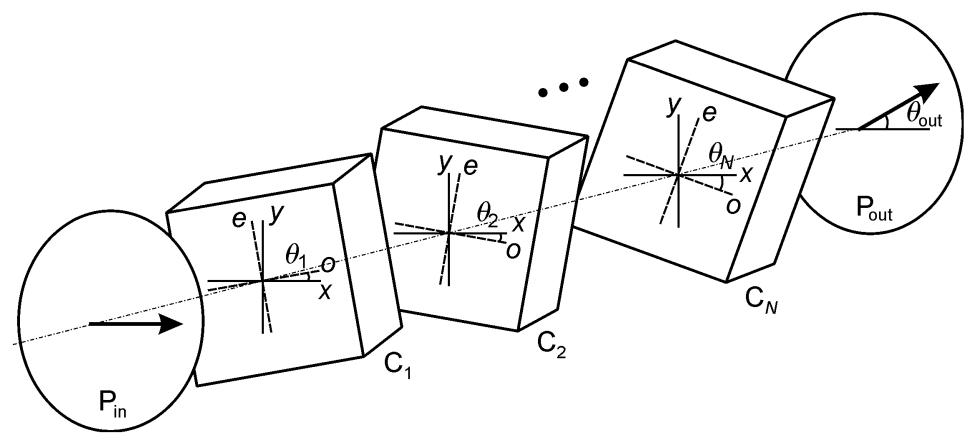

Fig. 9.28. Principle of a lattice filter based on birefringent crystals. Due to the refractive index difference for the ordinary $(o)$ and extraordinary $(e)$ waves, each crystal produces a differential delay of the fundamental polarisation components. The angular offsets between consecutive crystal's optic axes determine the coupling between the o and e waves from one crystal to the next. $P$ : polarizer, $C$ : crystal. After [47]

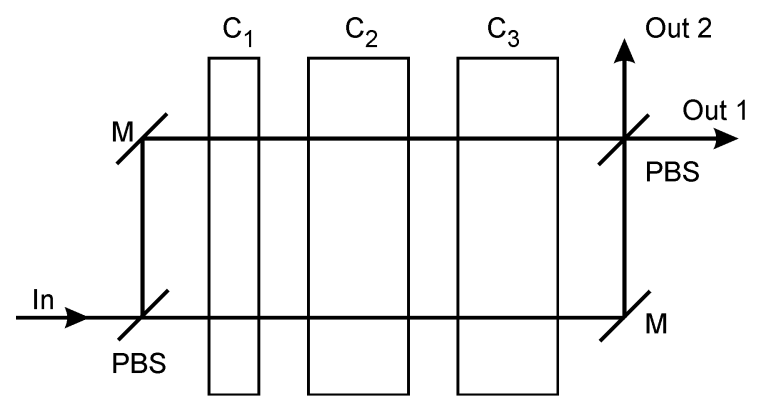

Fig. 9.29. Polarisation-independent birefringent crystal lattice filter using polarisation diversity. M: mirror; PBS: polarisation beam splitter; $\mathrm{C}_{i}$ : crystal $i$. After [45]

The same authors designed an electro-optically tuned interleaver as a variation of the structure of Fig. 9.30, where one of the blocks has been replaced by an electro-optic lithium niobate crystal. It requires about $4 \mathrm{kV}$ for tuning over its free spectral range [48].

Since the crystals or the glass blocks will usually need to be many thousands of wavelengths long, the effects of thermal expansion can be severe. Compensation of thermal drift can be obtained by applying carefully matched combinations of different crystals. Although lattice-type filters can be designed to have zero dispersion (see Sect. 9.4), it has also been proposed to use matched interleavers as multiplexers and demultiplexers, respectively, one having positive and the other negative group delay. An interleaver that can be changed to have positive or negative group delay by moving a half-waveplate to a different position is shown in Fig. 9.31 [49]. 


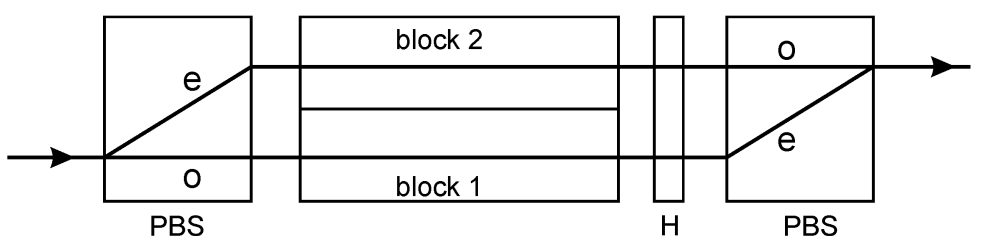

Fig. 9.30. Differential delay unit using "artificial" birefringence by providing separate propagation paths for the orthogonal polarisations through two blocks of different materials, after [46]. PBS: birefringent walk-off crystal serving as polarising beamsplitter; H: half-waveplate rotating the polarisation by 90 degrees

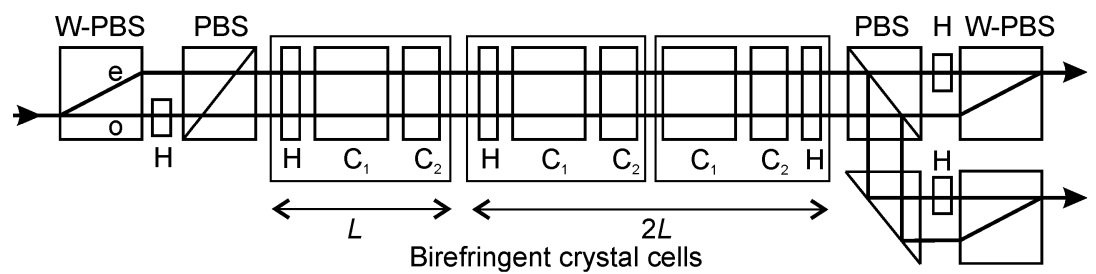

Fig. 9.31. Interleaver with selectable positive or negative group delay, after [49]. Selection is done by moving the input half-waveplate from the o-beam to the e-beam. WPBS: walk-off polarising beamsplitter; PBS: polarising beamsplitter; $\mathrm{H}$ : halfwaveplate; $\mathrm{C}_{1}, \mathrm{C}_{2}$ temperature-compensating birefringent crystals

\section{Michelson-Gires-Tournois Interferometers}

The equivalent of a ring resonator is the Fabry-Perot resonator in bulk optics. A Fabry-Perot resonator with one $100 \%$ mirror, which can only be used in reflection, is known as a Gires-Tournois resonator (GTR) [50], see also Chap. 6, Sect. 6.3.3. It acts as an allpass reflection filter having a periodic and strongly nonlinear phase response, just as the ring resonator coupled to a single waveguide does in transmission (Fig. 9.19).

Then, the bulk equivalent of the Mach-Zehnder interferometer with a ring resonator coupled to one of its branches is the Michelson interferometer (MI), where one or two of the mirrors of the MI are replaced by a GTR, thus forming a so-called Michelson-Gires-Tournois interferometer, see Fig. 9.32 and refs. [51, 52]. If both MI mirrors are replaced by GTR's, a more rectangular-shaped passband can be obtained [53]. Although one of the interleaver output signals will be back reflected, it can be separated from the input signal by tilting some of the mirrors. More complicated designs and several references to the patent literature can be found in [1].

A fibre analogue of a Michelson-Gires-Tournois interferometer has been demonstrated in [54]. 


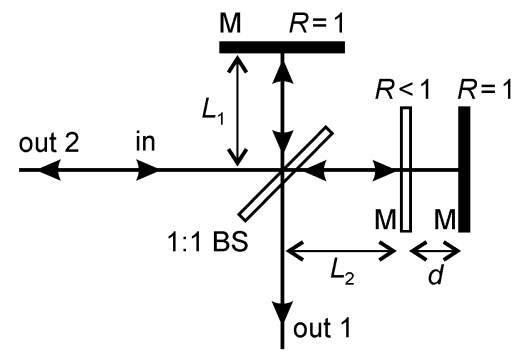

Fig. 9.32. Michelson-Gires-Tournois interferometer filter, after [52]. The back reflected light has a frequency response that is complementary to that of the transmitted light, and the input signal will appear frequency interleaved at the reflection and transmission port of the device

\section{Fabry-Perot Resonator Arrays}

Fabry-Perot (FP) resonators are discussed in Chap. 6. By arranging a number of such resonators in series, while carefully choosing the mirror reflectances, the rectangular-shaped transfer function that is desirable for interleaver operation can be well approximated, see the configuration of Fig. 9.33 [55].

This technique is not limited to conventional bulk FP etalons, but it can also be applied to the design of thin-film filters [56].

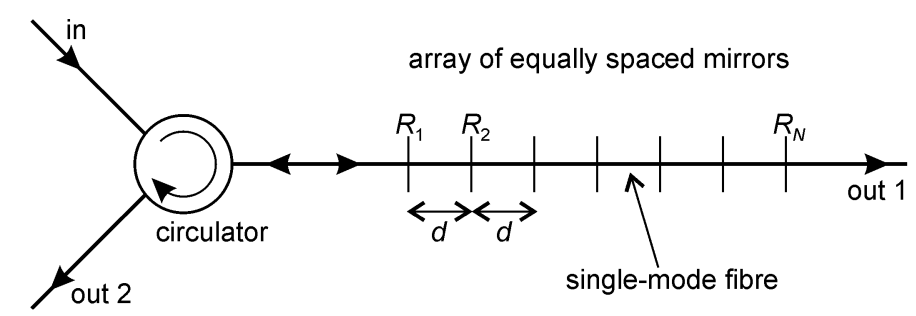

Fig. 9.33. Interleaver based on Fabry-Perot resonator arrays using a circulator for separating input and reflected output, after [55]. Outputs 1 and 2 are complementary, producing frequency-interleaved signals

\section{Fibre-based Interleavers}

Fibre Bragg gratings (FBG's) are very suitable for fabricating high-quality filter elements, see Chap. 5. Interleavers can be built by combining several equidistantly tuned FBG's, see e. g. [57]. The principle is shown in Fig. 9.34. 


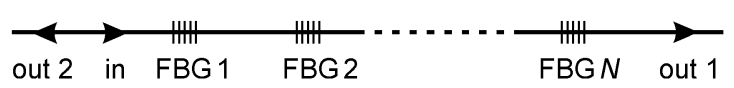

Fig. 9.34. Array of fibre Bragg gratings (FBG) [57]

As in Fig. 9.33, the reflected output can be separated from the input by using a circulator. A related technology is engineering a fibre Bragg grating in such a way, by sampling and chirping, that it reflects multiple channels $[25,58]$, which can be selected to provide interleaver functionality. This technique has been taken further by combining the fibre Bragg grating based FP resonators with a fibre MZI interleaver [59].

Besides the fibre Bragg-grating technology mentioned above, latticetype fibre MZI interleavers have been realised. Due to the relatively long fibre length of the branches - almost inevitable because of handling constraints -, inaccuracies of cutting fibre lengths, and thermal drift, tuning facilities and active stabilisation circuits are generally needed. An interesting approach using a reference light source with a wavelength outside the regular transmission band is shown in Fig. 9.35 [60, 61]. Other variations include a reflective fibre MZI using a loop mirror or a fibre Bragg grating [62], and a configuration using highly birefringent fibres [63]. Li et al. [64] exploited the interference between two modes in an $8 \mathrm{~cm}$ section of twomoded fibre for obtaining a compact thermally tuneable MZI-like filter with $1.72 \mathrm{~nm}$ FSR.

A $3 \times 3$ (or $1 \times 3$ ) interleaver based on three-arm fibre MZI filters, using symmetric $3 \times 3$ fused fibre directional couplers was investigated by Wang et al. [65]. Fewer of such filters would need to be cascaded for separating a given number of channels, compared to $2 \times 2$ interleavers. A $100 / 300 \mathrm{GHz}$ interleaver with $40 \mathrm{GHz}$ passband width at the $-0.5 \mathrm{~dB}$ level has been demonstrated. The same authors propose a three-armed MZI with allpass ringresonators coupled to each of the arms [66]. They show the design of

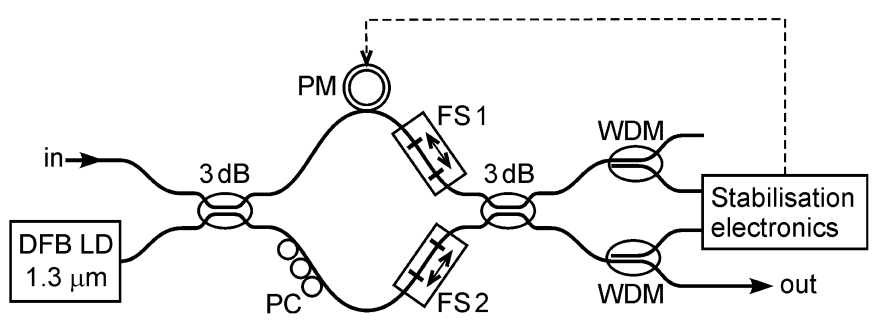

Fig. 9.35. Fibre MZI, stabilized using a reference light source. The filter can be tuned by changing the reference wavelength. DFB LD: distributed feedback laser diode, $3 \mathrm{~dB}$ : $3 \mathrm{~dB}$-directional coupler, PM: phase modulator (piezoelectric microstretcher), PC: polarisation controller, FS: fibre stretcher (mm range), WDM: coarse wavelength division multiplexing fibre coupler [61] 
a $100 / 300 \mathrm{GHz} 3 \times 3$ interleaver with a $85 \mathrm{GHz}$ passband width. However, the transmission curves of adjacent channels overlap somewhat in the transition region.

A binary tree cascade of fibre MZI's resulting in a 16-channel demultiplexer with $100 \mathrm{GHz}$ channel spacing has also been demonstrated [67].

\subsubsection{Planar Optical Waveguide Interleavers}

Most work on interleaver filters in planar technology has been done in silica-based waveguide systems, especially by AT\&T/Lucent $[4,33,15$, $21,68,69]$ and NTT [70, 7, 3, 71, 2], but also others [72]. Temperature dependence in such filters can be compensated by introducing short grooves filled with a material having a thermo-optic coefficient of the opposite sign as that of silica [73]. A clear trend in this technology is towards higher refractive index contrast in order to allow for smaller bend radii and hence more compact designs, as illustrated by the interleaver described in [74].

Stronger refractive index contrast can be obtained by using different materials, e.g. silicon oxynitride ( $\mathrm{SiON})[40,41]$. This technology has been used by IBM and the University of Twente for fabricating resonantcoupler based filters, and binary tree cascaded interleavers [75-77, 5].

Still higher contrasts are possible with semiconductor materials like GaAs [78] or silicon [79]. Although these materials certainly allow for more compact designs, they also put high demands on etching process quality because roughness will cause strong scattering loss due to the high contrast. Also, fibre-chip coupling becomes increasingly difficult as contrast increases.

Polymers can be attractive for these applications $[80,81]$ because of their simple processing compared to the inorganic materials mentioned before, and hence possibly lower cost. Also, they generally have an order of magnitude larger thermo-optic coefficient so that tuning power requirements can be lower. These materials may however have problems with long-term stability.

Lithium niobate is an attractive material because of its electro-optic properties and mature technology. Filters fabricated in this technology are often based on frequency-selective mode conversion, e. g. [82]. Electro-optically tuneable interleavers based on this principle are described in [83], Fig. 9.36.

Cusmai et al. [84] proposed a device based on a three-arm MZI, using three-way directional couplers instead of the usual $2 \times 2$ devices. Different from the device reported in [65], the power coupling ratio of the directional couplers is not 1:1:1 here. The device and its equivalent built from $2 \times 2$ MZI's are shown in Fig. 9.37. The $2 \times 2$ cascade may have the advantage that a better isolation can be obtained for a given fabrication accuracy. 


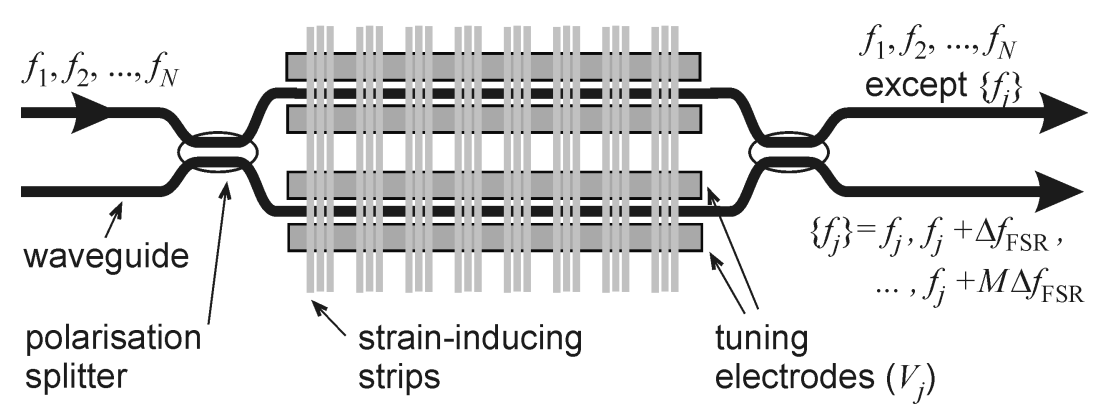

Fig. 9.36. Electro-optically tuneable lithium niobate interleaver [83]. The straininducing strips produce an off-diagonal element in the refractive index tensor leading to TE-TM mode conversion. The frequencies at which the phase matching condition for efficient mode conversion occurs is tuned by a voltage on the electrodes, changing the birefringence of the lithium niobate crystal

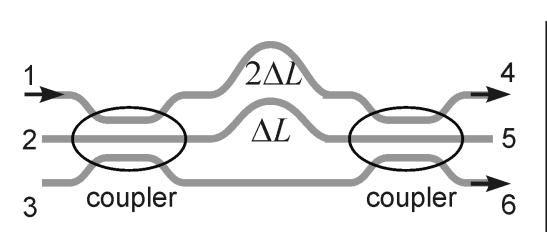

(a)

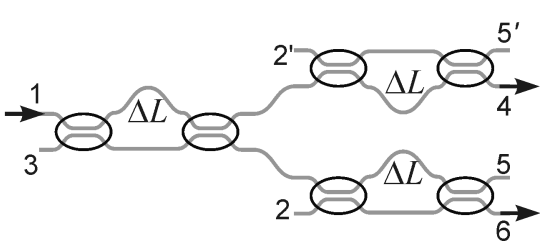

(b)

Fig. 9.37. (a) Three-arm MZI, and (b) its equivalent built from conventional two-arm MZI's (after [84]). The 3-way directional coupler is designed to have a 50:0:50 power splitting ratio from the central input channel to the respective output channels and a 25:50:25 ratio when excited at one of the outside channels. In normal operation, the 3 -arm MZI is applied as a $2 \times 2$ device, using only the outside input ( 1 and 3 ) and output ports (4 and 6)

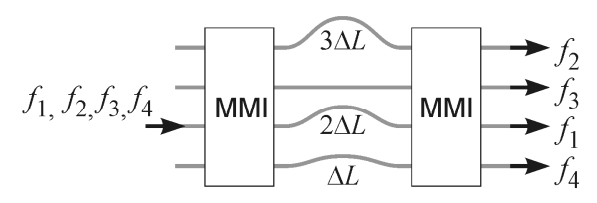

(a)

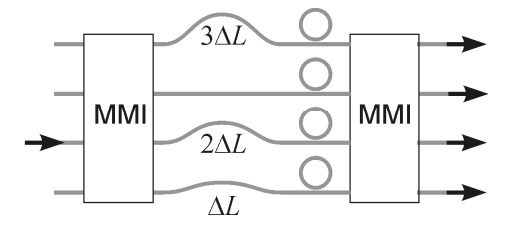

(b)

Fig. 9.38. Multiple-branch MZI interleavers (MMI: multi-mode interferometer). (a) Basic periodic $4 \times 4$ demultiplexer; for $(1 \times 4)$ interleaver functionality only a single input is used. (b) Improved transfer function by adding nonlinear phase shifters (ring resonators) to each branch (after [87])

As can be inferred from the equivalent circuit, the 3-arm MZI has the squared response of a single 2-arm MZI, thus providing a limited degree of passband flattening and stopband broadening. Compared to the 2-arm 
MZI, it also has reduced sensitivity to fabrication errors affecting the splitting ratio of the couplers. It should be noted that another approach by Oguma et al. [71] provides a method for obtaining highly accurate splitting ratios using a composition of inaccurate couplers and delay-lines.

Generalised multiple-branch MZI's have been introduced before, where multi-mode interferometers (MMI's) act as multi-port couplers (Fig. 9.38 (a)), e. g. van Dam et al. [85] and Lierstuen and Sudbø [86]. This type of device, shown in Fig. 9.38 for 4 input $\times 4$ output ports, can be generalised to $N \times N$ ports, and bears a strong similarity with the arrayed waveguide gratings (AWG's) that have been discussed in Chap. 4. MMI-based devices may be designed to have smaller insertion loss than AWG's, but they have fewer degrees of freedom for optimisation of the transfer functions. The concept was further optimised for passband flattening by Madsen [87], by introducing nonlinear phase shifters in each of the MZI branches (Fig. 9.38b), similar to the MZI + ring discussed in Sect. 9.3.2. Xiao and He recently discussed cross-talk reduction of MMI-based demultiplexers by cascading each of the output channels with a $1 \times 1$ multiple-branch MZI interferometer (consisting of a cascade of a $1 \times M$ and an $M \times 1 \mathrm{MMI}$, interconnected by $M$ waveguides providing the appropriate phase shifts) acting as a band-pass filter. They also modelled a $1 \times 16$ demultiplexer built from a binary-tree-type cascade of $1 \times 4$ interleavers [88].

In Sect. 9.1 the application of an interleaver for reducing the channel spacing and improving the passband shape of an AWG router by Oguma et al. [3] has already been mentioned. Although it is not an interleaver application in the strict sense, it is worth mentioning that Doerr et al. $[68,69]$ demonstrated significant passband flattening of an AWG by directly connecting the two output channels of the $3 \mathrm{~dB}$ output coupler of a singlestage MZI periodic filter to the AWG input coupler. The field distribution at the combined MZI output channels shifts periodically with frequency.

If the FSR of the MZI is equal to the AWG channel spacing, the combination of the Gaussian imaging properties of a basic AWG with the periodic shift of its input field leads to a passband-flattened overall response.

Photonic crystals [89] are an emerging technology for integrated optics. They promise extremely compact optical circuits, and their strong dispersive properties make them interesting for designing optical filters. As yet, the technology is still immature, the main problems being the optical loss and the fabrication accuracy of the sub-micron features of these structures. Fig. 9.39 shows a proposed interleaver which operates like an extremely compact two-mode interference device [90, 91]. 


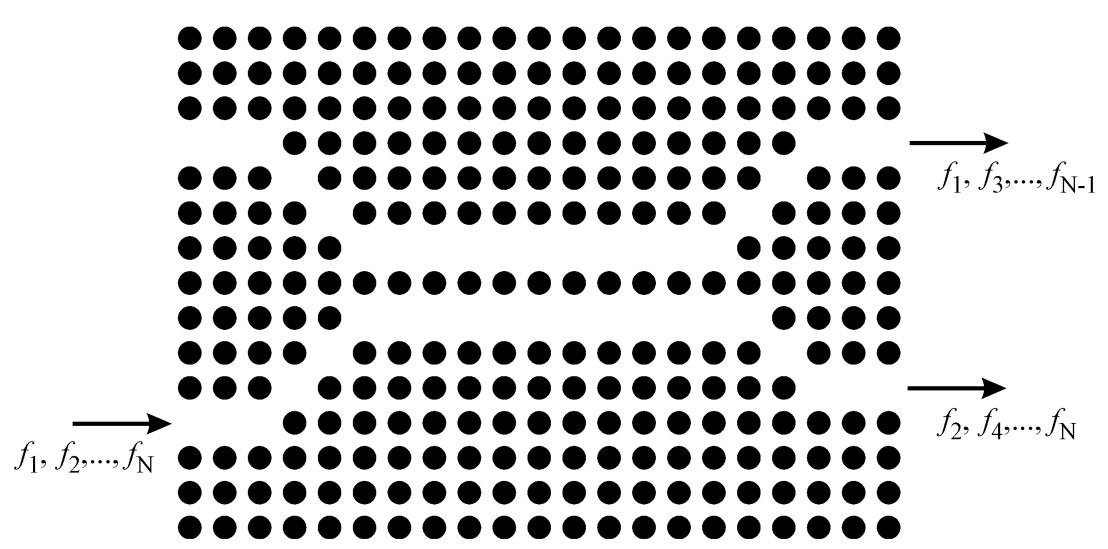

Fig. 9.39. Two coupled waveguides in a photonic crystal form a two-mode interferometer acting as an interleaver [90]

\subsubsection{Some Commercially Available Interleavers}

As can be seen from the references in the preceding sections, several large companies such as NTT, Lucent and IBM have investigated interleaver technologies. However, although these results may have been applied in proprietary systems, these companies do not seem to have marketed such interleavers as separately available products. Also, several interleaver patents have been assigned to other companies that do not seem to be active in this market; see e.g. the references in [1]. Most of the commercially available interleavers that were visible on the Internet in the period of June 2004 to May 2005 are represented in Table 9.1. More details about the suppliers and model designations are given in Table 9.2.

Most manufacturers do not supply detailed information on the technology that is used; no data was available on the specific designs. A distinguishing feature of these interleavers is the width of the passband that ranges from 25 to $68 \%$ of the free spectral range. 


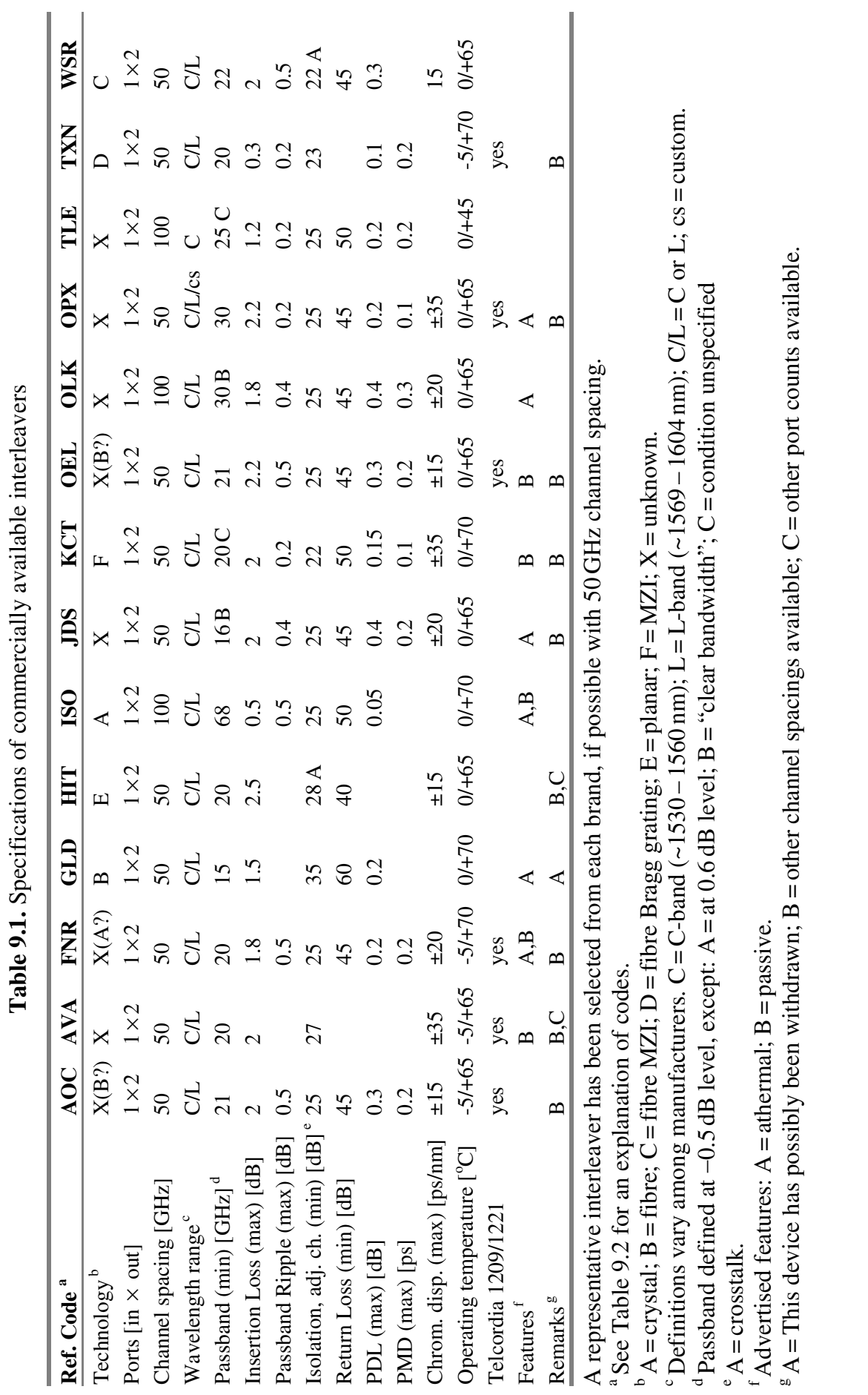




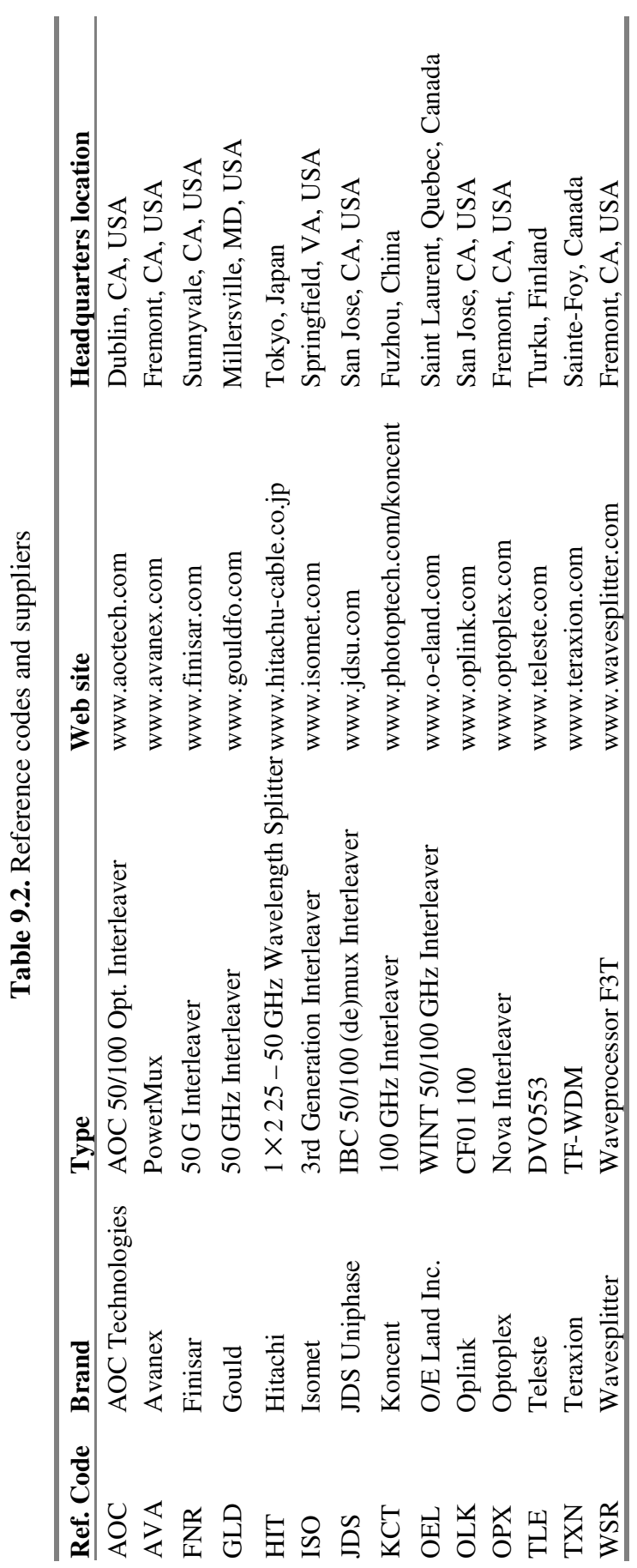




\subsection{Outlook}

Interleaver technology provides a route towards a cost-effective increase of bandwidth utilisation of optical fibre communication links. They can be used as add-on components for upgrading existing systems as well as for building blocks in innovative wavelength routers and multiplexers with a high channel count.

Although much effort has been devoted to optimising interleavers in bulk-type technologies, which has given rise to highly sophisticated devices, most progress is to be expected from planar waveguide technologies with their inherent potential for integration. This integration will enable the realisation of large cascades of interleavers, each of which may consist of many stages. Such composite devices allow the elimination of many fibre connections, thus promising increasing functionality, improved reliability and reduced insertion loss in a small package. In order to accommodate the desired functionality on a limited chip area, there is a clear trend towards increasing the integration density by using stronger refractive index contrast. This high contrast enables emerging VLSI photonics technologies like micro ring resonator arrays and photonic crystals. Planar technology also provides the potential for low-voltage and low-power electrical tuneability of the interleavers, using thermo-optic or electro-optic effects.

It should be emphasized that the resonant coupler approach, possibly in combination with ring-resonator based allpass filters, which has been shown in Sect. 9.3 for interleaver design, has a much wider applicability to filter design. For example, (gain) equalisers, dispersion compensators, and deconvolution filters may be designed using a very similar approach and may be realised in the same technology, thus leading to a cross-fertilisation among these fields.

If hitherto largely unused wavelength ranges in optical fibre communications (e.g. the range between the 1300 and $1500 \mathrm{~nm}$ windows) will be opened up, this will have a strong effect on the requirements for future interleaver technology. Depending on available optical amplifiers, a strong need may arise for banded interleavers (see Fig. 9.2c) having a very sharp roll-off characteristic, that can separate adjacent groups of wavelength channels. Alternatively, interleavers may be desired that operate in an extremely wide wavelength range of $1250-1650 \mathrm{~nm}$, separating more than 1000 channels. Such extreme requirements will really stress both fabrication technology and device design. 


\section{References}

1. S. Cao, J. Chen, J. N. Damask, C. R. Doerr, L. Guiziou, G. Harvey, Y. Hibino, H. Li, S. Suzuki, K.-Y.Wu, and P.Xie: "Interleaver technology: comparisons and applications requirements," J. Lightwave Technol. 22, 281-289 (2004)

2. T.Mizuno, T. Kitoh, M. Oguma, Y.Inoue, T. Shibata, and H. Takahashi: "MachZehnder interferometer with a uniform wavelength period," Opt. Lett. 29, 454456 (2004)

3. M. Oguma, T. Kitoh, K. Jinguji, T. Shibata, A. Himeno, and Y. Hibino: "Passbandwidth broadening design for WDM filter with lattice-form interleave filter and arrayed-waveguide gratings," IEEE Photon. Technol. Lett. 14, 328-330 (2002)

4. B.H. Verbeek, C.H. Henry, N. A. Olsson, K. J. Orlowsky, R. F. Kazarinov, and B. H. Johnson: "Integrated four-channel Mach-Zehnder multi/demultiplexer fabricated with phosphorous doped $\mathrm{SiO}_{2}$ waveguides on $\mathrm{Si}$," J. Lightwave Technol. 6, 1011-1015 (1988)

5. C. G. H. Roeloffzen, F. Horst, B. J. Offrein, R. Germann, G. L. Bona, H. W. M. Salemink, and R. M.de Ridder: "Tunable passband flattened 1-from-16 binary-tree structured add-after-drop multiplexer using $\mathrm{SiON}$ waveguide technology," IEEE Photon. Technol. Lett. 12, 1201-1203 (2000)

6. C. Kostrzewa and K. Petermann: "Bandwidth optimization of optical add/drop multiplexers using cascaded couplers and Mach-Zehnder sections," IEEE Photon. Technol. Lett. 7, 902-904 (1995)

7. K. Jinguji, N. Takato, Y.Hida, T. Kitoh, and M. Kawachi: "Two-port optical wavelength circuits composed of cascaded Mach-Zehnder interferometers with pointsymmetrical configurations," J. Lightwave Technol. 14, 2301-2310 (1996)

8. M. Sharma, H. Ibe, and T. Ozeki: "Optical lattice-type add-drop multiplexing filters and their use in WDM networks," Opt. Fiber Technol. 4, 117-134 (1998)

9. T.Liu, Y.C.Soh, Y.Zhang, and Z. Fang: "Parameter optimization of an all-fiber Fourier filter flat-top interleaver," Opt. Eng. 41, 3217-3220 (2002)

10. G. Cincotti: "Fiber wavelet filters," IEEE J. Quantum Electron. 38, 1420-1427 (2002)

11. Q.Wang and S.He: "Optimal design of a flat-top interleaver based on cascaded M-Z interferometers by using a genetic algorithm," Opt. Commun. 224, 229-236 (2003)

12. Q.J.Wang, T.Liu, Y.C.Soh, and Y.Zhang: “All-fiber Fourier filter flat-top interleaver design with specified performance parameters," Opt. Eng. 42, 3172-3178 (2003)

13. S. W. Kok, Y.Zhang, C. Wen, and Y.C. Soh: "Design of all-fiber optical interleavers with a given specification on passband ripples," Opt. Commun. 226, 241-248 (2003)

14. T. Zhang, K. Chen, and Q. Sheng: "A novel interleaver based on dual-pass MachZehnder interferometer," Microw. Opt. Technol. Lett. 42, 253-255 (2004)

15. C.K. Madsen and J.H.Zhao: "A general planar waveguide autoregressive optical filter,” J. Lightwave Technol. 14, 437-447 (1996)

16. O. Schwelb: "Characteristics of lattice networks and spectral filters built with $2 \times 2$ couplers,” J. Lightwave Technol. 17, 1470-1480 (1999) 
17. O. Schwelb: "Transmission, group delay, and dispersion in single-ring optical resonators and add/drop filters - A tutorial overview," J. Lightwave Technol. 22, 1380-1394 (2004)

18. K. Jinguji and M. Oguma: "Optical half-band filters,” J. Lightwave Technol. 18, 252-259 (2000)

19. C. J.Kaalund and G.D.Peng: "Pole-zero diagram approach to the design of ring resonator-based filters for photonic applications," J. Lightwave Technol. 22, 1548-1559 (2004)

20. M. M. Spühler and D. Erni: "Towards structural optimization of planar integrated lightwave circuits," Opt. Quantum Electron. 32, 701-718 (2000)

21. C.K.Madsen and J.H.Zhao: "Postfabrication optimization of an autoregressive planar waveguide lattice filter," Appl. Opt. 36, 642-647 (1997)

22. C.K.Madsen: "Efficient architectures for exactly realizing optical filters with optimum bandpass designs," IEEE Photon. Technol. Lett. 10, 1136-1138 (1998)

23. G. Cincotti and A. Neri: "Logarithmic wavelength demultiplexers," J. Lightwave Technol. 21, 1576-1583 (2003)

24. G. Cincotti and A. Neri: "Design and performances of logarithmic wavelength demultiplexers," IEEE Photon. Technol. Lett. 16, 1325-1327 (2004)

25. R. Slavik and S. LaRochelle: "Large-band periodic filters for DWDM using multiple-superimposed fiber Bragg gratings," IEEE Photon. Technol. Lett. 14, 1704$1706(2002)$

26. G. Lenz, B. J.Eggleton, C. R. Giles, C. K. Madsen, and R. E. Slusher: "Dispersive properties of optical filters for WDM systems," IEEE J. Quantum Electron. 34, 1390-1402 (1998)

27. C. K. Madsen and J.H.Zhao: Optical Filter Design and Analysis, a signal processing approach (John Wiley \& Sons, New York, 1999)

28. A.V.Oppenheim and R.W.Schafer: Digital Signal Processing (Prentice-Hall, Englewood Cliffs, NJ, 1975)

29. S. K. Mitra: Digital signal processing: a computer-based approach, $2^{\text {nd }}$ edition (McGraw-Hill, New Delhi, 2001)

30. J.G. Proakis and D.G. Manolakis: Digital signal processing: principles, algorithms, and applications, $2^{\text {nd }}$ edition (Macmillan Publishing, New York, 1992)

31. B. Moslehi, J.W. Goodman, M.Tur, and H.J.Shaw: "Fiber-optic lattice signal processing," Proc. IEEE 72, 909-930 (1984)

32. M. Kuznetsov: "Cascaded coupler Mach-Zehnder channel dropping filters for wavelength-division-multiplexed optical systems," J. Lightwave Technol. 12, 226-230 (1994)

33. H.H. Yaffe, C.H.Henry, M. H. Serbin, and L. G. Cohen: "Resonant couplers acting as add-drop filters made with silica-on-silicon waveguide technology," J. Lightwave Technol. 12, 1010-1014 (1994)

34. K. Jinguji and M. Kawachi: "Synthesis of coherent two-port lattice-form optical delay-line circuit," J. Lightwave Technol. 13, 73-82 (1995)

35. K. Oda, N. Takato, H. Toba, and K. Nosu: "A wide-band guided-wave periodic multi/demultiplexer with a ring resonator for optical FDM transmission systems", J. Lightwave Technol. 6, 1016-1023 (1988)

36. S. Suzuki, M. Yanagisawa, Y. Hibino, and K. Oda, "High-density integrated planar lightwave circuits using $\mathrm{SiO}_{2}-\mathrm{GeO}_{2}$ waveguides with a high refractive index difference," J. Lightwave Technol. 12, 790-796 (1994) 
37. M. Kohtoku, S.Oku, Y. Kadota, Y. Shibata, and Y. Yoshikuni: "200-GHz FSR periodic multi/demultiplexer with flattened transmission and rejection band by using a Mach-Zehnder interferometer with a ring resonator," IEEE Photon. Technol. Lett. 12, 1174-1176 (2000)

38. C. K. Madsen: "General IIR optical filter design for WDM applications using allpass filters," J. Lightwave Technol. 18, 860-868 (2000)

39. N. Takato, A. Sugita, K. Onose, H. Okazaki, M. Okuno, M. Kawachi, and K. Oda: "128-Channel polarization-insensitive frequency-selection-switch using highsilica waveguides on Si," IEEE Photon. Technol. Lett. 2, 441-443 (1990)

40. K. Wörhoff, P. V.Lambeck, and A. Driessen: "Design, tolerance analysis and fabrication of silicon oxynitride based planar optical waveguides for communication devices," J. Lightwave Technol. 17, 1401-1407 (1999)

41. R. Germann, H.W. M. Salemink, R. Beyeler, G. L. Bona, F. Horst, I. Massarek, and B.J.Offrein: "Silicon oxynitride layers for optical waveguide applications," J. Electrochem. Soc. 147, 2237-2241 (2000)

42. C. Vázquez, S.E. Vargas, J.M. S. Pena, and A.B. Gozalo, "Demultiplexers for ultranarrow channel spacing based on Mach-Zehnders and ring resonators," Opt. Eng. 43, 2080-2086 (2004)

43. B. Lyot: "Un monochromateur à grand champ utilisant les interférences en lumière polarisée," C. R. Acad. Sci. (Paris) 197, 1593-1595 (1933)

44. I. Šolc: "Birefringent chain filters," J. Opt. Soc. Amer. 55, 621-625, (1965)

45. W.J.Carlsen and C.F.Buhrer, "Flat passband birefringent wavelength-division multiplexers," Electron. Lett. 23, 106-107 (1987)

46. J.Zhang, L. Liu, and Y.Zhou: "A tunable interleaver filter based on analog birefringent units," Opt. Commun. 227, 283-294 (2003)

47. J.Zhang, L. Liu, and Y.Zhou: "Novel and simple approach for designing latticeform interleaver filter," Opt. Express 11, 2217-2224 (2003)

48. J.Zhang, L. Liu, and Y.Zhou: "Optimum design of a novel electro-optically tunable birefringent interleaver filter," J. Opt. A: Pure Appl. Opt. 6, 1052-1057 (2004)

49. J.Chen: "Dispersion-compensating optical digital filters for $40-\mathrm{Gb} / \mathrm{s}$ metro adddrop applications," IEEE Photon. Technol. Lett. 16, 1310-1312 (2004)

50. F. Gires and P. Tournois: "Interféromètre utilisable pour la compression d'impulsions lumineuses modulées en fréquence," C. R. Acad. Sci. 258, 6112 6115, (1964)

51. B. B. Dingel and M. Izutsu: "Multifunction optical filter with a Michelson-GiresTournois interferometer for wavelength-division-multiplexed network system applications," Opt. Lett. 23, 1099-1101 (1998)

52. B.B.Dingel and T.Aruga: "Properties of a novel noncascaded type, easy-todesign, ripple-free optical bandpass filter," J. Lightwave Technol. 17, 1461-1469 (1999)

53. C.H.Hsieh, R. Wang, Z. J.Wen, I. McMichael, P. Yeh, C. W.Lee, and W.H.Cheng: "Flat-top interleavers using two Gires-Tournois etalons as phase-dispersive mirrors in a Michelson interferometer," IEEE Photon. Technol. Lett. 15, 242-244 (2003)

54. Q.J.Wang, Y.Zhang, and Y.C.Soh: "Efficient structure for optical interleavers using superimposed chirped fiber Bragg gratings," IEEE Photon. Technol. Lett. 17, 387-389 (2005) 
55. S. Yim and H.F. Taylor: "Spectral slicing optical waveguide filters for dense wavelength division multiplexing," Opt. Commun. 233, 113-117 (2004)

56. H.Chen, P. Gu, Y.Zhang, M. Ai, W.Lv, and B. Jin: "Analysis on the match of the reflectivity of the multi-cavity thin film interleaver," Opt. Commun. 236, 335-341 (2004)

57. J. Kim, J.Park, S.Chung, N. Park, B. Lee, and K. Jeong: "Bidirectional wavelength add/drop multiplexer using two separate MUX and DEMUX pairs and reflectiontype comb filters," Opt. Commun. 205, 321-327 (2002)

58. H. Lee and G.P. Agrawal: "Add-drop multiplexers and interleavers with broadband chromatic dispersion compensation based on purely phase-sampled fiber gratings," IEEE Photon. Technol. Lett. 16, 635-637 (2004)

59. R. Slavík and S. LaRochelle: "All-fiber periodic filters for DWDM using a cascade of FIR and IIR lattice filters," IEEE Photon. Technol. Lett. 16, 497-499 (2004)

60. J. T. Ahn, H. K. Lee, K. H. Kim, M. Y. Jeon, D. S. Lim, and E. H. Lee: "A stabilised fibre-optic Mach-Zehnder interferometer filter using an independent stabilisation light source," Opt. Commun. 157, 62-66 (1998)

61. J.T. Ahn, H. K. Lee, M. Y. Jeon, D. S. Lim, and K. H. Kim: "Continuously tunable multi-wavelength transmission filter based on a stabilised fibre-optic interferometer," Opt. Commun. 165, 33-37 (1999)

62. H. Yonglin, L. Jie, M.Xiurong, K. Guiyun, Y.Shuzhong, and D.Xiaoyi: "High extinction ratio Mach-Zehnder interferometer filter and implementation of singlechannel optical switch," Opt. Commun. 222, 191-195 (2003)

63. Y.Lai, W.Zhang, J.A.R. Williams, and I. Bennion: "Bidirectional nonreciprocal wavelength-interleaving coherent fiber transversal filter," IEEE Photon. Technol. Lett. 16, 500-502 (2004)

64. Q. Li, C.-H.Lin, and H.P.Lee: "A novel electrically tuned all-fiber comb filter," Opt. Fiber Commun. Conf. (OFC'04) Techn. Digest (Los Angeles, CA, USA, 2004) Vol. 1, 23-27 (2004)

65. Q. J. Wang, Y.Zhang, and Y.C. Soh: "All-fiber 3x3 interleaver design with flat-top passband," IEEE Photon. Technol. Lett. 16, 168-170 (2004)

66. Q.J.Wang, Y.Zhang, and Y.C.Soh: "Design of 100/300 GHz optical interleaver with IIR architectures,” Opt. Express 13, 2643-2652 (2005)

67. C.H.Huang, H. Luo, S.Xu, and P.Chen: "Ultra-low loss, temperature-insensitive 16-channel 100-GHz dense wavelength division multiplexers based on cascaded all-fiber unbalanced Mach-Zehnder structure," Opt. Fiber Commun. Conf. (OFC'99), Techn. Digest (San Diego, CA, USA, 1999) Vol. 1, 79-81 (1999)

68. C. R. Doerr, L. W. Stulz, R. Pafchek, and S. Shunk, "Compact and low-loss manner of waveguide grating router passband flattening and demonstration in a 64channel blocker/multiplexer," IEEE Photon. Technol. Lett. 14, 56-58 (2002)

69. C. R. Doerr, L. W. Stulz, and R. Pafchek: "Compact and low-loss integrated boxlike passband multiplexer,” IEEE Photon. Technol. Lett. 15, 918-920 (2003)

70. N. Takato, K. Jinguji, M. Yasu, H. Toba, and M. Kawachi: "Silica-based singlemode waveguides on silicon and their application to guided-wave optical interferometers," J. Lightwave Technol. 6, 1003-1010 (1988)

71. M. Oguma, T. Kitoh, Y. Inoue, T. Mizuno, T. Shibata, M. Kohtoku, and Y. Hibino: "Compact and low-loss interleave filter employing lattice-form structure and silica-based waveguide,” J. Lightwave Technol. 22, 895-902 (2004) 
72. D. Di Mola, G.Sanvito, M.Lenzi, and E. Fioravanti: "Flat-band add-drop FIR lattice filter design,” IEEE J. Select. Topics Quantum Electron. 5, 1366-1372 (1999)

73. S. Kamei, M. Oguma, M. Kohtoku, T. Shibata, and Y. Inoue: "Low-loss athermal silica-based lattice-form interleave filter with silicone-filled grooves," IEEE Photon. Technol. Lett. 17, 798-800 (2005)

74. T. Mizuno, Y.Hida, T. Kitoh, M. Kohtoku, M. Oguma, Y. Inoue, and Y. Hibino: "12.5-GHz spacing compact and low-loss interleave filter using $1.5 \% \Delta$ silicabased waveguide," IEEE Photon. Technol. Lett. 16, 2484-2486 (2004)

75. B. J. Offrein, G. L. Bona, F. Horst, H. W. M. Salemink, R. Beyeler, and R. Germann: "Wavelength tunable optical add-after-drop filter with flat passband for WDM networks," IEEE Photon. Technol. Lett. 11, 239-241 (1999)

76. B. J. Offrein, R. Germann, F. Horst, H. W. M. Salemink, R. Beyeler, and G. L. Bona: "Resonant coupler-based tunable add-after-drop filter in silicon-oxynitride technology for WDM networks," IEEE J. Select. Topics Quantum Electron., 5, 14001406 (1999)

77. B. J. Offrein, F. Horst, G. L. Bona, H.W. M. Salemink, R. Germann, and R. Beyeler: "Wavelength tunable 1-from-16 and flat passband 1-from-8 add-drop filters," IEEE Photon. Technol. Lett. 11, 1440-1442 (1999)

78. M. H. Hu, Z.Huang, K. L. Hall, R. Scarmozzino, and R. M. Osgood: "An integrated two-stage cascaded Mach-Zehnder device in GaAs," J. Lightwave Technol. 16, 1447-1455 (1998)

79. Y. J.Lin, S. L. Lee, and C.L. Yao: "Four-channel coarse-wavelength division multiplexing demultiplexer with a modified Mach-Zehnder interferometer configuration on a silicon-on-insulator waveguide," Appl. Opt. 42, 2689-2694 (2003)

80. C. Kostrzewa, R. Moosburger, G. Fischbeck, B. Schüppert, and K. Petermann: "Tunable polymer optical add/drop filter for multiwavelength networks," IEEE Photon. Technol. Lett. 9, 1487-1489 (1997)

81. B. Chen, H. Jia, J.Zhou, D. Zhao, H. Lu, Y. Yuan, and M. Iso: "Optimized design of fluorinated polyimide based interleaver," Appl. Opt. 42, 4202-4207 (2003)

82. P.Tang, O.Eknoyan, and H.F. Taylor: "Rapidly tunable polarisation independent optical add-drop multiplexer in Ti: $\mathrm{LiNbO}_{3}$," Electron. Lett. 38, 242-244 (2002)

83. H.F. Taylor: "Tunable spectral slicing filters for dense wavelength-division multiplexing," J. Lightwave Technol. 21, 837-847 (2003)

84. G. Cusmai, F. Morichetti, R. Costa, A. Melloni, and M. Martinelli: "An integrated optical interleaver based on a three-arms Mach-Zehnder interferometer," Proc. $12^{\text {th }}$ Europ. Conf. Integr. Optics (ECIO'05), Grenoble, France, pp. 418-421 (2005)

85. C. van Dam, M.R.Amersfoort, G. M.ten Kate, F.P. G. M.van Ham, M. K. Smit, P. A. Besse, M. Bachmann, and H. Melchior: "Novel InP-based phased-array wavelength demultiplexer using a generalized MMI-MZI configuration," Proc. $7^{\text {th }}$ Europ. Conf. Integr. Optics (ECIO'95), Delft, The Netherlands, pp. 275-278 (1995)

86. L. O. Lierstuen and A. Sudbø, "8-Channel wavelength division multiplexer based on multimode interference couplers," IEEE Photon.Technol. Lett. 7, 1034-1036 (1995)

87. C. K. Madsen: “A multiport frequency band selector with inherently low loss, flat passbands, and low crosstalk," IEEE Photon. Technol. Lett. 10, 1766-1768 (1998) 
88. Y.Xiao and S.He, "An MMI-based demultiplexer with reduced cross-talk," Opt. Commun. 247, 335-339 (2005)

89. J.D. Joannopoulos, R. D. Meade, and J. N. Winn: Photonic crystals: molding the flow of light (Princeton University Press, Princeton, NJ, USA, 1995)

90. S. Boscolo, M. Midrio, and C.G. Someda: "Coupling and decoupling of electromagnetic waves in parallel 2-D photonic crystal waveguides," IEEE J. Quantum Electron. 38, 47-53 (2002)

91. J.Zimmermann, M. Kamp, A. Forchel, and R. März: "Photonic crystal waveguide directional couplers as wavelength selective optical filters," Opt. Commun. 230, 387-392 (2004) 